\title{
Parallel Coding of First- and Second-Order Stimulus Attributes by Midbrain Electrosensory Neurons
}

\author{
Patrick McGillivray, ${ }^{1}$ Katrin Vonderschen, ${ }^{1}$ Eric S. Fortune, ${ }^{2,3,4}$ and Maurice J. Chacron ${ }^{1,5}$ \\ ${ }^{1}$ Department of Physiology, McGill University, Montreal, Quebec H3G 1Y6, Canada, ${ }^{2}$ Department of Psychological and Brain Sciences, Johns Hopkins \\ University, Baltimore, Maryland 21218, ${ }^{3}$ Department of Neuroscience, Johns Hopkins University, Baltimore, Maryland 21205, ${ }^{4}$ Museum of Zoology, \\ Pontificia Universidad Católica del Ecuador, Quito, Ecuador, and 5 Department of Physics, McGill University, Montreal, Quebec H3A 2T8, Canada
}

\begin{abstract}
Natural stimuli often have time-varying first-order (i.e., mean) and second-order (i.e., variance) attributes that each carry critical information for perception and can vary independently over orders of magnitude. Experiments have shown that sensory systems continuously adapt their responses based on changes in each of these attributes. This adaptation creates ambiguity in the neural code as multiple stimuli may elicit the same neural response. While parallel processing of first- and second-order attributes by separate neural pathways is sufficient to remove this ambiguity, the existence of such pathways and the neural circuits that mediate their emergence have not been uncovered to date. We recorded the responses of midbrain electrosensory neurons in the weakly electric fish Apteronotus leptorhynchus to stimuli with first- and second-order attributes that varied independently in time. We found three distinct groups of midbrain neurons: the first group responded to both first- and second-order attributes, the second group responded selectively to first-order attributes, and the last group responded selectively to second-order attributes. In contrast, all afferent hindbrain neurons responded to both first- and second-order attributes. Using computational analyses, we show how inputs from a heterogeneous population of $\mathrm{ON}$ - and OFF-type afferent neurons are combined to give rise to response selectivity to either first- or second-order stimulus attributes in midbrain neurons. Our study thus uncovers, for the first time, generic and widely applicable mechanisms by which parallel processing of first- and second-order stimulus attributes emerges in the brain.
\end{abstract}

\section{Introduction}

Natural sensory stimuli often have rich spatiotemporal structure characterized by first- and second-order attributes that vary independently of one another (Attias and Schreiner, 1997; Simoncelli and Olshausen, 2001; Lewicki, 2002; Heil, 2003; Joris et al., 2004; Mante et al., 2005; de Kock and Sakmann, 2009). For auditory stimuli, first- and second-order attributes would correspond to acoustic pressure and amplitude, respectively.

Although second-order attributes are critical for perception (Shannon et al., 1995, 1998; Bertoncini et al., 2009), their neural processing continues to pose a challenge to investigators. This is in part because sensory neurons adapt their response properties in response to changes in both first- and second-order attributes (Brenner et al., 2000; Fairhall et al., 2001; Nagel and Doupe, 2006; Maravall et al., 2007; Lundstrom et al., 2008, 2010). This process optimizes coding (Barlow, 1961; Laughlin, 1989; Wark et al., 2007) but also creates ambiguity as the same neural response (e.g., firing rate) can then be elicited by multiple stimuli with different attributes (Fairhall et al., 2001; Wark et al., 2007).

Received Feb. 1, 2012; revised Feb. 21, 2012; accepted Feb. $28,2012$.

Author contributions: M.J.C. designed research; P.M., K.V., and M.J.C. performed research; P.M. and M.J.C. analyzed data; P.M., E.S.F., and M.J.C. wrote the paper.

This research was supported by the Canadian Institutes of Health Research, the Natural Sciences and Engineering Research Council of Canada, the Canada Foundation for Innovation, and the Canada Research Chairs (M.J.C.).

Correspondence should be addressed to Maurice J. Chacron, 3655 Sir William Osler, Room 1137, Montreal, QC H3G 1Y6, Canada. E-mail: maurice.chacron@mcgill.ca.

DOI:10.1523/JNEUROSCI.0478-12.2012

Copyright $\odot 2012$ the authors $\quad 0270-6474 / 12 / 325510-15 \$ 15.00 / 0$
One solution to this problem is to have independent coding of first- and second-order attributes by separate and parallel neural pathways (Baker, 1999). Parallel processing of sensory information is a common strategy used across modalities including auditory (Takahashi et al., 1984; Oertel, 1999; Gelfand, 2004), visual (Marr, 1982; Livingstone and Hubel, 1987; Merigan and Maunsell, 1993), and electrosensory (Carr and Maler, 1986; Bell and Maler, 2005; Kawasaki, 2005), to code for different stimulus attributes. However, the pathways that encode first- and secondorder stimulus attributes and their associated neural circuitry remain poorly understood to this day.

Weakly electric fish are a well suited model system for understanding the coding of first- and second-order stimulus attributes because of well characterized natural stimuli and neural pathways devoted to their processing (Chacron et al., 2011). Peripheral electroreceptor neurons project onto pyramidal cells within the hindbrain electrosensory lateral line lobe (ELL), which in turn project to the midbrain torus semicircularis (TS) (Carr and Maler, 1985; Bastian et al., 2004). In general, TS neurons display more response selectivity than ELL neurons (Chacron et al., 2009; Chacron and Fortune, 2010; Khosravi-Hashemi et al., 2011; Vonderschen and Chacron, 2011), which is consistent with the emergence of a sparse neural code (Chacron et al., 2011). While it was previously found that electroreceptors and ELL pyramidal cells respond to both first- and second-order attributes of electrosensory stimuli (Middleton et al., 2006; Savard et al., 2011), the responses of TS neurons to second-order attributes have not been previously described. We found that a distinct subpopula- 
tion of TS neurons selectively responds to second-order attributes while another subpopulation selectively responds to firstorder attributes and thus provide the first experimental evidence for the emergence of parallel processing of first- and secondorder stimulus attributes by distinct neural pathways. Through a combination of computational analysis and mathematical modeling, we reveal simple physiological neural circuits that can implement such parallel coding.

\section{Materials and Methods}

Animal housing and surgery. The weakly electric fish species Apteronotus leptorhynchus was used in this study. Animals of either sex were obtained from tropical fish suppliers and acclimated to the laboratory according to published guidelines (Hitschfeld et al., 2009). For experiments, animals were immobilized by intramuscular injection of Tubocurarine chloride hydrate ( $1 \mu \mathrm{g} / \mathrm{g}$ body weight; Sigma) and respirated via a mouth tube with aerated tank water at a flow rate of $\sim 10 \mathrm{ml} / \mathrm{min}$. The fish was submerged in water except for the top of the head. To expose the brain for recording, we first locally anesthetized the skin on the skull by applying $2 \%$ Lidocaine. Then we removed $\sim 6 \mathrm{~mm}^{2}$ of skin to expose the skull to which a metal post was glued for stabilization. By drilling a hole of $\sim 2$ $\mathrm{mm}^{2}$ through the skull, we gained access to the area of the tectum overlying the TS, or to the eminentia granularis posterior overlying the ELL. The surface of the brain was kept covered by saline (Bastian, 1974) throughout the experiment. Details have been described previously (Bastian et al., 2002; Chacron et al., 2005c, 2007; Chacron and Bastian, 2008; Krahe et al., 2008; Toporikova and Chacron, 2009; Savard et al., 2011). McGill University's animal care and use committee approved all procedures.

Recording. Extracellular recordings were obtained from both the ELL and TS brain regions of Apteronotus leptorhynchus using either glass micropipettes or Woods metal electrodes (Rose and Fortune, 1996; Chacron et al., 2009; Chacron and Fortune, 2010; Khosravi-Hashemi et al., 2011; Vonderschen and Chacron, 2011). Histological analysis of recording sites has shown that our TS recordings were obtained from most layers (Vonderschen and Chacron, 2011). Recordings from ELL pyramidal neurons were obtained from the centromedial, centrolateral, and lateral segments. Pyramidal cells within all three segments can be distinguished based on the mediolateral and rostrocaudal positions of the recording electrode with respect to surface landmarks such as the "T0" vein and its afferent veins (Maler et al., 1991), the depth at which recordings are obtained, as well as their responses to sensory input as previously described (Krahe et al., 2008). As pyramidal cells within all three segments did not differ in their responses to the noise stimuli used in this study when using the measures described below, the data from all three ELL segments were pooled. The electrode signal was amplified (Axoclamp 2B, Axon Instruments or Model 1000 amplifier, A-M Systems) and digitized at $10 \mathrm{kHz}$ using a CED Power1401 with Spike2 software.

Stimulation. Because Apteronotus leptorhynchus has a neurogenic electric organ, the electric organ discharge (EOD) persists after curare injection. All stimuli used in this study consisted of amplitude modulations (AMs) of the animal's own EOD. These were delivered using standard techniques (Chacron et al., 2003; Krahe et al., 2008). The fish's EOD was recorded with chloridized silver wire electrodes positioned at the head and at the tail. The zero crossings of the amplified EOD signal (DAM50, World Precision Instruments; bandpass filter between $300 \mathrm{~Hz}$ and $3 \mathrm{kHz}$ ) were detected by a window discriminator, which then triggered a function generator to output a single-cycle sinusoid of slightly higher frequency than the fish's EOD. This created a train of single-cycle sinusoids that were phase-locked to the EOD. The train was then multiplied (MT3 multiplier, Tucker Davis Technologies) with an AM waveform. The resulting signal was attenuated (LAT45 attenuator, Leader Electronics) and fed into the experimental tank via a stimulus isolator (A395 linear stimulus isolator, World Precision Instruments) and was delivered by a set of two electrodes located $\sim 20 \mathrm{~cm}$ on each side of the fish. Note that this stimulation geometry is referred to as "global" in previous studies, as the AMs of the EOD will impinge upon a large portion of the animal's electrosensory epithelium (Krahe et al., 2008). Note that, as the signal is added to the animal's own EOD, the signal led to an increase or a decrease in amplitude of the EOD depending on its polarity compared with that of the EOD.

It is essential to recognize that the EOD waveform itself, which is nearly sinusoidal, can be considered as a carrier signal and that the meaningful stimulus here is the AM of this carrier signal. For this reason, we will henceforth refer to the AM waveform as the stimulus $S(t)$. We will be considering first- and second-order attributes of the stimulus $S(t)$ itself as described below. We note that these correspond to the second- and thirdorder attributes of the full signal that is received by the animal (i.e., the AM multiplied with the EOD waveform mimic), respectively.

The stimuli used in this study consisted of (1) sinusoidal waveforms with frequency $4 \mathrm{~Hz}$, (2) low-pass filtered zero-mean broadband (eighthorder Butterworth filter, $120 \mathrm{~Hz}$ cutoff) and, (3) bandpass filtered zero-mean narrowband (fourth-order Butterworth, 40-60 Hz band) Gaussian white noise. The noise stimuli mimic the sensory input from two or more neighboring fish (Middleton et al., 2006, 2011; Stamper et al., 2010). The mean stimulus contrast (i.e., the ratio of the SD of the $\mathrm{AM}$ caused by the stimulus divided by the EOD baseline amplitude) were $10-20 \%$, which is similar to what was used in previous studies (Bastian et al., 2002, 2004; Chacron et al., 2005b; Toporikova and Chacron, 2009). The $40-60 \mathrm{~Hz}$ and $0-120 \mathrm{~Hz}$ noise stimuli each lasted $20 \mathrm{~s}$ and were each presented 5 times (Chacron, 2006; Krahe et al., 2008; Avila-Akerberg et al., 2010) to quantify response variability as described below. The $4 \mathrm{~Hz}$ sinusoidal AM was presented only once and lasted for at least $20 \mathrm{~s}$.

Data analysis. All data analysis was performed using MATLAB (The MathWorks). The recordings were first high-pass filtered using an eighth-order Butterworth filter with a $300 \mathrm{~Hz}$ cutoff and the spike times were obtained as the times at which the signal crosses a suitably chosen threshold from below. A binary sequence with binwidth $d t=0.5 \mathrm{~ms}$ was then created using the following rule: the content of bin $i$ was set to 1 if there is an action potential at time $t_{i}$ where $i^{\star} d t<t_{i} \leq(i+1)^{\star} d t$ and to 0 otherwise. Note that, as $d t$ is smaller than the absolute refractory period of ELL and TS neurons (typically 1-2 ms), there can be at most one spike occurring during any given bin. We subsequently refer to this binary sequence as the response $R(t)$.

Firing rate. The firing rate was computed during baseline (i.e., in the absence of stimulation or $S(t)=0$ ) activity that lasted at least $20 \mathrm{~s}$.

Phase histogram in response to $4 \mathrm{~Hz}$ sinusoidal stimulation. The spike times $t_{i}$ accumulated in response to sinusoidal stimulation were converted into phases $\Phi_{i}$ with phase 0 defined to be at the maximum of the sinusoidal stimulus. We then built phase histograms with binwidth 0.25 radians. We then computed the bimodality index in the following manner. First, we performed a circular permutation of the phase histogram such that the bin with maximum content is now located at 0 (note that this is equivalent to subtracting the phase at which the bin count is maximum from the phase sequence $\Phi_{i}$ and then recomputing the phase histogram from this new sequence). The bimodality index was then obtained by dividing the bin count at phase $\pi$ by the bin count at phase 0 . As such, neurons that tend to respond more or less equally near two phases of the stimulus separated by $\pi$ radians are characterized by a bimodality index near 1 whereas neurons that respond preferentially near a given phase of the sinusoidal stimulus are instead characterized by bimodality indices near 0 .

Coherence. We used the coherence measure to characterize ELL and TS neurons' responses to first-order attributes of the $40-60 \mathrm{~Hz}$ and $0-120$ $\mathrm{Hz}$ noise stimuli used in this study. The responses to the 5 repeated presentations of the stimulus waveform $S(t)$ were labeled $R_{1}(t) \ldots R_{5}(t)$. We computed the stimulus-response coherence using:

$$
C_{R S}(f)=\frac{\left|\frac{1}{5} \sum_{i=1}^{5} P_{R_{i} S}(f)\right|^{2}}{\frac{P_{S S}(f)}{5} \sum_{i=1}^{5} P_{R_{i} R_{i}}(f)}
$$




$$
R(t)=\sum_{i=1}^{5} R_{i}(t)
$$

where $P_{R_{i} S}(f)$ is the cross-spectrum between $R_{i}(t)$ and $S(t), P_{R_{i} R_{i}}(f)$ is the power spectrum of $R_{i}(t)$, and $P_{S S}(f)$ is the power spectrum of $S(t)$.

We also computed the response-response coherence between the responses to repeated presentations of the same stimulus as (Chacron, 2006):

$$
C_{R R}(f)=\frac{\left|\frac{1}{10} \sum_{i=2}^{5} \sum_{j=1}^{i-1} P_{R_{i} R_{j}}(f)\right|^{2}}{\left[\frac{1}{5} \sum_{i=1}^{5} P_{R_{i} R_{i}}(f)\right]^{2}} .
$$

Unlike the stimulus-response coherence, the response-response coherence is only limited by the trial-to-trial variability in the neural response to repeated presentations of the same stimulus and we always have $C_{R S}(f) \leq\left[C_{R R}(f)\right]^{1 / 2}$ (Roddey et al., 2000). Thus, to compare the two measures, we always plotted the square root of the response-response coherence but refer to it simply as the response-response coherence for brevity. Since the response-response coherence gives an upper bound on the amount of information that can be transmitted (Roddey et al., 2000), the normalized first-order response was computed as the average of the maximum value of the stimulus-response coherence divided by the maximum value of the response-response coherence for $0-120 \mathrm{~Hz}$ and $40-60 \mathrm{~Hz}$ noise stimuli. Note that the response-response coherence can be non-zero at frequencies outside those found in the stimulus. We note that an analysis of our data using the integral of the coherence over the range $0-300 \mathrm{~Hz}$ rather than the maximum value yielded similar results (data not shown).

Envelopes. We also quantified whether our neurons in our data were also responsive to the second-order attributes of the stimulus $S(t)$. Thus, we computed the envelope $E(t)$ of the stimulus $S(t)$. Simply speaking, the envelope $E(t)$ can be thought of as the instantaneous amplitude of the stimulus $S(t)$ and is thus a second-order attribute of the stimulus waveform $S(t)$ as it is related to variance. In the case of the narrowband $40-60 \mathrm{~Hz}$ noise stimulus, it corresponds to the smooth line that connects successive maxima in the stimulus waveform. In general, the envelope $E(t)$ can be obtained from the stimulus $S(t)$ by the following nonlinear transformation:

$$
E(t)=\sqrt{S(t)^{2}+\hat{S}(t)^{2}}
$$

where $\hat{S}(t)$ is the Hilbert transform of $S(t)$ (Myers et al., 2003; Middleton et al., 2006; Savard et al., 2011). It is given by:

$$
\hat{S}(t)=\frac{1}{\pi} C\left[\int_{-\infty}^{+\infty} \frac{S(\tau)}{t-\tau} d \tau\right],
$$

where $C$ is the Cauchy principal value. We note that the time-varying envelope of $40-60 \mathrm{~Hz}$ and $0-120 \mathrm{~Hz}$ noise stimuli is a consequence of the filtering process. This is because unfiltered Gaussian white noise is characterized by a flat (i.e., constant) envelope,

We quantified responses to the envelope by computing the coherence between the envelope $E(t)$ and the response $R(t)$, the envelope-response coherence $C_{E R}(f)$, given by:

$$
C_{E R}(f)=\frac{\left|\frac{1}{5} \sum_{i=1}^{5} P_{R_{i} E}(f)\right|^{2}}{\frac{P_{E E}(f)}{5} \sum_{i=1}^{5} P_{R_{i} R_{i}}(f)}
$$

where $P_{E E}(f)$ is the power spectrum of $E(t)$ and $P_{R_{i} E}(f)$ is the crossspectrum between $R_{i}(t)$ and $E(t)$. The normalized second-order response was computed as the average between the maximum value of the envelope-response coherence divided by the maximum value of the responseresponse coherence for $0-120 \mathrm{~Hz}$ and $40-60 \mathrm{~Hz}$ noise stimuli.

Second-order selectivity index. The second-order selectivity index was obtained for each neuron by dividing the normalized second-order response by the normalized first-order response and by taking the logarithm in base 10 of that ratio. As such, negative values imply that the normalized first-order response is greater than the normalized second response while positive values imply the opposite.

Phase histograms and rectification in response to noise stimulation. Phase histograms in response to either $40-60 \mathrm{~Hz}$ or $0-120 \mathrm{~Hz}$ noise stimuli were computed by extracting the instantaneous phase of the stimulus which is given by:

$$
\varphi(t)=\arctan \left[\frac{\hat{S}(t)}{S(t)}\right] .
$$

We then built a histogram of the sequence of phases $\left[\varphi\left(t_{i}\right)\right]$ that correspond to the spike times $\left[t_{i}\right]$ with binwidth 0.1 radians.

We considered a neuron to display phase locking only when the onset of action potential firing occurred reliably at a given phase (Keener et al., 1981; Trussell, 1999; Chacron et al., 2004). As such, neurons that reliably display nonlinear rectification (i.e., are driven into cessation of firing) at some stimulus phases must then reliably fire action potentials at other stimulus phases and thus also display phase locking (Savard et al., 2011). Phase locking is thus a nonlinear phenomenon that gives rise to peaks in the spike train power spectrum at integer multiples (i.e., higher harmonics) of the frequencies contained in the stimulus waveform (i.e., the fundamental frequencies) (Ewert et al., 2008; Savard et al., 2011; Schneider et al., 2011). We therefore quantified phase locking by computing the ratio of the power at the second harmonic (i.e., 3 times the fundamental frequencies) to that at the fundamental frequencies as done previously (Schneider et al., 2011). Specifically, for $40-60 \mathrm{~Hz}$ noise stimulation, we obtained a phase locking index by dividing the value of the spike train power spectrum at $150 \mathrm{~Hz}$ by its value at $50 \mathrm{~Hz}$. For $0-120 \mathrm{~Hz}$ noise stimulation, the value of the spike train power spectrum at $300 \mathrm{~Hz}$ was divided by the value at $100 \mathrm{~Hz}$ to obtain the phase locking index. The average of both values was then taken for each neuron. Finally, the set of phase locking index values obtained for our dataset was normalized by its maximum value.

We note that other measures that can be used to quantify phase locking such as vector strength (Mardia and Jupp, 1999) can be nonzero even when the relationship between the stimulus waveform and the neuronal output firing rate is linear in nature (Schneider et al., 2011), a situation that does not give rise either to phase locking as defined above or nonlinear rectification, and are thus not appropriate in this case.

Ascertaining whether a neuron responded to the stimuli. A given neuron was deemed to respond to the stimuli presented in this study if the maximum value of the response-response coherence was $>0.1$.

Spike-triggered average. Spike-triggered averages (STAs) were obtained by averaging the stimulus waveform within a $50 \mathrm{~ms}$ time window surrounding recording spikes. The STA is defined by:

$$
\operatorname{STA}(t)=\frac{1}{N} \sum_{i=1}^{N} S\left(t+t_{i}\right),
$$

where the average is performed over the $N$ spike times $\left[t_{i}\right]$.

E-vs I-type responses in ELL and TS. We quantified the phase between the neural response $R(t)$ and the stimulus waveform $S(t)$ as:

$$
\phi(f)=\arctan \left(\frac{\operatorname{imag}\left[P_{R S}(f)\right]}{\operatorname{real}\left[P_{R S}(f)\right]}\right),
$$

where $\operatorname{imag}(.$.$) and \operatorname{real}(\ldots)$ are the imaginary and real parts, respectively, and $P_{R S}(f)$ is the cross-spectrum between $R(t)$ and $S(t)$. A neuron was deemed to be E type (i.e., excited by an increase in the 
stimulus waveform $S(t))$ if $-\pi / 4 \leq \phi(0) \leq \pi / 4$ and I type (i.e., inhibited by an increase in the stimulus waveform $S(t))$ if $3 \pi / 4 \leq$ $\phi(0) \leq \pi$ or $-\pi \leq \phi(0) \leq-3 \pi / 4$. We also found that the mean value of the STA, $\langle S T A(t)\rangle_{t}$, was negative for I-type neurons and positive for E-type neurons.

Spike-triggered covariance. STA analysis focuses on the ensemble of all stimulus segments directly preceding each spike to estimate which stimulus feature, on average, is most likely to elicit a spike. In contrast, spiketriggered covariance (STC) analysis focuses on the variability within the ensemble of stimulus segments preceding each spike and is used to gain a more detailed picture of the (potentially) multiple stimulus features that give rise to spiking activity (Brenner et al., 2000; Agüera y Arcas et al., 2003; Slee et al., 2005; Fairhall et al., 2006; Gussin et al., 2007; Gollisch and Meister, 2008).

We accumulated stimulus segments up to $50 \mathrm{~ms}$ before each spike time $t_{i}$. Since the stimulus was sampled at $d t=0.5 \mathrm{~ms}$, each segment contained 100 elements. We then computed the covariance matrix $\mathbf{C}$ whose element $j k(1 \leq j, k \leq 100)$ is given by:

$$
\begin{aligned}
C_{j k}=\frac{1}{N} \sum_{i=1}^{N}\left[S\left(-j d t+t_{i}\right)-S T A(-j d t)\right][S( & \left.k d t+t_{i}\right) \\
& -S T A(-k d t)],
\end{aligned}
$$

where $t_{i}$ are the spike times. Because we are interested in the stimulus features that cause spiking, we subtracted the prior distribution of stimuli, $C^{\text {prior }}$ (which is Gaussian along all stimulus dimensions, independent of the response), from the covariance matrix $C$, to obtain a matrix representing the covariance differences (Brenner et al., 2000; Fairhall et al., 2006):

$$
\hat{C}_{j k}=C_{j k}-C_{j k}^{\text {prior }} .
$$

The covariance matrix of the prior $C^{\text {prior }}$ is simply the covariance matrix of the stimulus waveform $S(t)$ itself and represents the variability that is due to the stimulus waveform alone:

$$
\begin{array}{r}
C_{j k}^{\text {prior }}=\frac{1}{M} \sum_{i=1}^{M}[S(-j d t+i d t)-S T A(-j d t)][S(-k d t+i d t) \\
-S T A(-k d t)]
\end{array}
$$

where the stimulus $S(t)$ is assumed to start at $t=0$ and end at $t=M$ $d t$. We estimated $C^{\text {prior }}$ by taking random $50 \mathrm{~ms}$ long segments of $S(t)$ without reference to spiking as done previously (Brenner et al., 2000).

We diagonalized $\hat{C}$ to find its eigenvalues $\hat{\lambda}_{i}$ and corresponding eigenvectors $\hat{v}_{i}=\left(\hat{v}_{i}(-50 d t) \ldots \hat{v}_{i}(0)\right)(1 \leq i \leq 100)$. The eigenvalues were sorted in ascending order (i.e., $\hat{\lambda}_{i} \leq \hat{\lambda}_{i+1}$ ). Because the prior stimulus distribution was subtracted from the covariance matrix, an eigenvalue of zero corresponds to the prior variance and indicates that the corresponding stimulus feature was not relevant to cell spiking. As such, we needed to only keep the eigenvalues that were significantly different from zero. A methodology has been proposed to assess whether a given eigenvalue differs significantly from zero (Agüera y Arcas et al., 2003; Fairhall et al., 2006; Gollisch and Meister, 2008). It is based on the fact that the eigenvalues of the matrix $\hat{C}$ depend on the number of spikes that are used in the analysis. Indeed, the eigenvalues that are not associated with spiking will decay to zero as a power law while those that are will remain stable when increasing number of spikes are used (Agüera y Arcas and Fairhall, 2003; Fairhall et al., 2006). However, to see this effect, large numbers (i.e., $\sim 10^{6}$ ) of spikes are needed, which makes such a methodology applicable only in modeling studies where it is possible to obtain arbitrarily large number of spikes (Agüera y Arcas et al., 2003) or in experimental studies with very long $(>12 \mathrm{~h}$ ) recording times (Fairhall et al., 2006).

Because it is not currently feasible to obtain such a large number of spikes from ELL and TS neurons experimentally, we could not use Agüera y Arcas et al.'s (2003) methodology here. Instead, we used a criterion is based on the fact that neurons typically have a finite integration time window (i.e., that only the stimulus waveform up to a certain time in the past can influence spiking at present). Since the stimulus waveform at times outside of the integration time window cannot influence spiking, the stimulus features associated with spiking will show high variability within the neuron's integration window and low variability elsewhere. As such, the variability in the stimulus segments from $-T_{\text {in }}$ to 0 was compared with the variability from -2 $T_{\text {in }}$ to $-T_{\text {in }}$, where $T_{\text {in }}$ is the integration time window. If the variability within $-T_{\text {in }}$ to 0 is comparable to that within $-2 T_{\text {in }}$ to $-T_{\text {in }}$, then the corresponding stimulus feature is most likely not associated with spiking. In contrast, if the variability within $-T_{\text {in }}$ to 0 is much greater than that within $-2 T_{\text {in }}$ to $-T_{\text {in }}$, then the corresponding stimulus feature is most likely associated with spiking.

The integration time window $T_{\text {in }}$ can be estimated from the STA as the maximum time before the action potential for which the STA is approximately equal to the mean value of the stimulus (zero in our case) (Agüera y Arcas et al., 2003; Svirskis et al., 2003; Bair and Movshon, 2004). We found empirically that the STAs for neurons in our dataset were all approximately constant up to $25 \mathrm{~ms}$ before the spike (see below). Thus, we took $T_{\text {in }}=25 \mathrm{~ms}$. To determine which eigenvalues $\hat{\lambda}_{m}$ of $\hat{C}$ were significantly different from 0 , we computed the set of projections of each stimulus segment on the eigenvector $\hat{v}_{m}$ associated with the eigenvalue $\hat{\lambda}_{m}$ as:

$P_{i, m}^{1}=\left[\hat{v}_{m}(-50 d t) \ldots \hat{v}_{m}(-25 d t)\right] \cdot\left[S\left(-50 d t+t_{i}\right) \ldots S\left(-25 d t+t_{i}\right)\right]$

$P_{i, m}^{2}=\left[\hat{v}_{m}(-25 d t) \ldots \hat{v}_{m}(0)\right] \cdot\left[S\left(-25 d t+t_{i}\right) \ldots S\left(t_{i}\right)\right]$,

where ". " is the dot product and $\left[t_{i}\right]$ are the spike times. We then computed the ratio:

$$
R A_{m}=\frac{\operatorname{STD}\left(P_{i, m}^{1}\right)}{\operatorname{STD}\left(P_{i, m}^{2}\right)},
$$

where $\operatorname{STD}(\ldots)$ is the $\mathrm{SD}$ computed over the spike times $\left[t_{i}\right]$.We found that in most (95\%) of cases, one eigenvalue $\hat{\lambda}_{h}$ was associated with a ratio $\mathrm{RA}_{\mathrm{h}}$ that was much larger (at least twice) than all others which were $\sim 1$ in value. We found empirically that the eigenvalue $\hat{\lambda}_{h}$ was always the eigenvalue that was greatest in magnitude. Thus, we assumed that the corresponding stimulus feature was the primary stimulus feature that was associated with spiking in neurons in our dataset and was retained for further analysis. A negative value for $\hat{\lambda}_{h}$ indicates an associated stimulus direction with decreased variability compared with the variability of the stimulus. This reduced variability indicates that stimuli eliciting a spike are well stereotyped along this direction. A positive eigenvalue is associated with a stimulus direction with greater variability than expected from the stimulus alone and indicates that multiple stimulus features cause spiking activity. We thus quantified the relative contributions of each feature by computing the projections:

$$
P_{i, h}=\left[\hat{v}_{m}\left(-50 d t+t_{i}\right) \ldots \hat{v}_{m}(0)\right] \cdot\left[S\left(-50 d t+t_{i}\right) \ldots S\left(t_{i}\right)\right] .
$$

The fraction of spike times $f_{\mathrm{E}}$ for which $P_{i, h}$ was positive was then determined and a bias index was then computed as $2 f_{\mathrm{E}}-1$. A negative value for the bias index implies that the stimulus segments that elicit spiking are negative on average. A positive value of the bias index implies the opposite. A bias index near zero indicates that approximately half of the stimulus segments that elicit spiking are positive while the other half is negative. The $\mathrm{E}$ and I filters were then computed as the weighted averages of the spike-triggered stimulus segments over the indices $k$ for which $P_{k, h}$ was positive and negative, with weights given by $f_{\mathrm{E}}$ and $1-f_{\mathrm{E}}$, respectively.

Modeling convergent E- and I-type input unto TS neurons. We built a model of convergent inputs from both E- and I-type ELL pyramidal neurons unto a single TS neuron. Both E- and I-type ELL pyramidal 
neurons were modeled using the linear leaky integrate-and-fire neuron (Lapicque, 1907) and were described by the following set of differential equations:

$$
\begin{aligned}
& \frac{d V_{E}}{d t}=-\frac{V_{E}}{\tau_{E}}+I_{\text {bias }, E}+S(t)+\xi_{E}(t) \\
& \frac{d V_{I}}{d t}=-\frac{V_{I}}{\tau_{I}}+I_{\text {bias }, I}-S(t)+\xi_{I}(t) \\
& V_{E}(t) \geq \theta_{E} \rightarrow V_{E}\left(t^{+}\right)=0 \\
& V_{I}(t) \geq \theta_{I} \rightarrow V_{I}\left(t^{+}\right)=0,
\end{aligned}
$$

where $V_{E}, V_{I}$ are the membrane voltages of the E- and I-type model neurons, respectively. $\tau_{\mathrm{E}}$ and $\tau_{\mathrm{I}}$ are the membrane time constants. $I_{\mathrm{bias}, E}$ and $I_{\text {bias }, I}$ are bias currents, $S(t)$ is the stimulus, and $\xi_{\mathrm{E}}(t), \xi_{\mathrm{I}}(t)$ are independent and identically distributed Gaussian white noise processes with zero mean and SDs $\sigma_{\mathrm{E}}$ and $\sigma_{\mathrm{I}}$, respectively. Since previous studies have found that E- and I-type ELL pyramidal neurons are excited and inhibited by the stimulus, respectively (Saunders and Bastian, 1984), we assumed that our model E- and I-type pyramidal cell receive stimulation waveforms that have opposite polarities, respectively, which is consistent with experimental results showing that their activities are out of phase with one another (Bastian et al., 2002). When $V_{j}(t)$ is greater than or equal to the threshold $\theta_{j}(j=\mathrm{E}, \mathrm{I}), V_{j}$ is immediately reset to 0 and maintained there for the duration of the absolute refractory period $T_{R, j}$ and a spike is said to have occurred at time $t$. As in the experimental data, the stimulus $S(t)$ was either $0-120 \mathrm{~Hz}$ low-pass filtered (eighth-order Butterworth) or $40-60 \mathrm{~Hz}$ bandpass filtered (fourth-order Butterworth) white noise with zero mean and SD 0.2.

The TS neuron is also modeled using a linear leaky integrate-and-fire model:

$$
\begin{gathered}
\frac{d V}{d t}=-\frac{V}{\tau}+I_{\text {bias, }}+\xi(t)+A \rho_{E} \sum_{k} \Theta\left(t-t_{E, k}\right) \alpha\left(t-t_{E, k}\right) \\
+A\left(1-\rho_{E}\right) \sum_{l} \Theta\left(t-t_{I, l}\right) \alpha\left(t-t_{I, l}\right) \\
V(t) \geq \theta \rightarrow V\left(t^{+}\right)=0,
\end{gathered}
$$

where $V$ is the membrane voltage, $\tau$ is the membrane time constant, $I_{\text {bias }}$ is a bias current, $\xi(t)$ is a Gaussian white noise process with zero mean and SD $\sigma, A$ is a constant, $0 \leq \rho_{\mathrm{E}} \leq 1$ is the fraction of E-type input received by the model TS neuron, and $\left[t_{E, k}\right]$ and $\left[t_{I, l}\right]$ are the spike times of the model E- and I-type ELL pyramidal neurons, respectively. Here $\Theta(t)$ is the Heaviside function $(\Theta(t)=1$ if $t \geq 0$ and $\Theta(t)=0$ otherwise), while $\alpha(t)$ is the $\alpha$ function with time constant $\tau_{\alpha}$ given by:

$$
\alpha(t)=\frac{t}{\tau_{\alpha}^{2}} \exp \left[-\frac{t}{\tau_{\alpha}}\right],
$$

when $V(t)$ is greater or equal than the threshold $\theta, V$ is immediately reset to 0 and maintained there during the duration of the absolute refractory period $T_{R}$ and a spike is said to have occurred at time $t$. The model was simulated numerically using an Euler-Maruyama integration algorithm (Kloeden and Platen, 1999) with integration time step $0.025 \mathrm{~ms}$. The spike times of the model TS neuron $\left[t_{j}\right]$ were accumulated and were then analyzed in the same manner as the experimental data as described above. Parameter values used for the simulations were $\tau_{\mathrm{E}}=\tau_{\mathrm{I}}=1 \mathrm{~ms}$, $I_{\text {bias }, E}=\mathrm{I}_{\text {bias }, I}=0.92, \sigma_{\mathrm{E}}=\sigma_{\mathrm{I}}=0.15, \theta_{E}=\theta_{I}=1.4, T_{R, E}=T_{R, I}=T_{R}=$ $2 \mathrm{~ms}, \tau=10 \mathrm{~ms}, I_{\text {bias }}=0.8, \sigma=0.8, \tau_{\alpha}=15 \mathrm{~ms}, \theta=15.5$, and $A=1.2$. These parameter values were chosen based on available experimental data (membrane and EPSP time constants, absolute refractory periods) (Fortune and Rose, 1997a; Toporikova and Chacron, 2009) or were adjusted such as to give firing rates and responses to the stimulus that were comparable to those seen experimentally (bias currents, noise and stimulus SDs, thresholds). In some simulations, $I_{\text {bias }, E}$ and $I_{\text {bias }, I}$ were both systematically covaried (i.e., $I_{\text {bias }, E}=I_{\text {bias }, I}$ ) between 0.82 and 1.25 to vary the firing rates of the model ELL pyramidal neurons.

\section{Results}

We made extracellular recordings from 66 ELL pyramidal neurons and from 125 TS neurons (Fig. $1 A$ ). The mean baseline (i.e., no stimulus) firing rate of ELL neurons was $19.48 \pm 1.56 \mathrm{~Hz}$, which is comparable to that obtained in previous studies using both intracellular and extracellular recordings (Bastian et al., 2002; Chacron et al., 2005b; Chacron, 2006; Krahe et al., 2008; Toporikova and Chacron, 2009; Avila-Akerberg et al., 2010) and much lower than the mean baseline firing rate of electroreceptor afferents: $199 \pm 8.1 \mathrm{~Hz}$ (Chacron et al., 2005a; Gussin et al., 2007). Further, the mean baseline firing rate of TS neurons was $5.45 \pm 0.71 \mathrm{~Hz}$, which is comparable to that obtained from intracellular recordings in previous studies (Vonderschen and Chacron, 2011) and is furthermore significantly lower than that of ELL neurons $\left(p<10^{-3}\right.$, Wilcoxon rank sum test, $\left.\mathrm{df}=190\right)$.

We used two categories of sensory stimuli: broadband $(0-120$ $\mathrm{Hz}$ ) (Fig. $1 B$, top) or narrowband $(40-60 \mathrm{~Hz})$ (Fig. $1 B$, bottom) noise. Both categories are behavioral relevant, as they are similar to naturally occurring signals generated by groups of conspecifics in the wild (Stamper et al., 2010). In particular, when two fish come into contact (i.e., are within $1 \mathrm{~m}$ of one another), each animal will experience an amplitude modulation of its own electric signal (i.e., a beat) that oscillates at the difference between the electric signal frequencies (Benda et al., 2005; Hupé and Lewis, 2008). We will henceforth refer to this amplitude modulation as the stimulus as it is directly encoded by electroreceptor afferents located on the fish's skin (Scheich et al., 1973; Bastian, 1981). However, the depth of modulation of the EOD, which is a second-order attribute as it is related to variance, can also vary dynamically in time when the distance between the two fish changes or when 3 or more fish are in close proximity (Middleton et al., 2006; Stamper et al., 2010). The depth of modulation is also known as the "envelope." Electrosensory envelopes are generally restricted to a low temporal frequency range, below $\sim 50 \mathrm{~Hz}$ (Stamper et al., 2010), whereas the stimulus typically contains temporal frequencies between 0 and $400 \mathrm{~Hz}$.

Both categories of sensory input differ dramatically in the relationship between the stimulus waveform (a first-order attribute) and its envelope (a second-order attribute), which is a result of the filtering process. For narrowband $40-60 \mathrm{~Hz}$ noise, the stimulus waveform and its envelope contain power in different frequency ranges: $40-60 \mathrm{~Hz}$ and $0-20 \mathrm{~Hz}$, respectively (Fig. $1 \mathrm{~B}$, blue, green). In other words, their spectral frequency contents are nonoverlapping (Fig. $1 C)$. The situation is quite different for the broadband $0-120 \mathrm{~Hz}$ noise, as the envelope's frequency content largely overlaps with that of the stimulus waveform (Fig. $1 B, C$ ). Also note that the stimulus waveform and its envelope are not correlated, that is to say that they are independent of each other, for both categories of sensory input.

We characterized both ELL and TS neural responses using three coherence measures. First, we computed the coherence between the stimulus waveform and the response (Fig. 1D). The coherence is a number between 0 and 1 that quantifies how linearly correlated the stimulus and response are at a given frequency, and thus provides a measure of the response to first-order stimulus attributes such as its time-varying value. Second, we computed the coherence between the envelope of the stimulus and the response, which provides a measure of the response to second-order stimulus attributes (Fig. $1 D)$. Third, we computed the coherence between responses to repeated presentations of the stimulus, which provides a measure of response reliability at different frequencies (Fig. 1D). Since the response-response coherence is always greater than or equal to either the stimulus-response or the envelope-response coherence (Roddey et al., 2000), we divided the stimulus-response and envelo- 

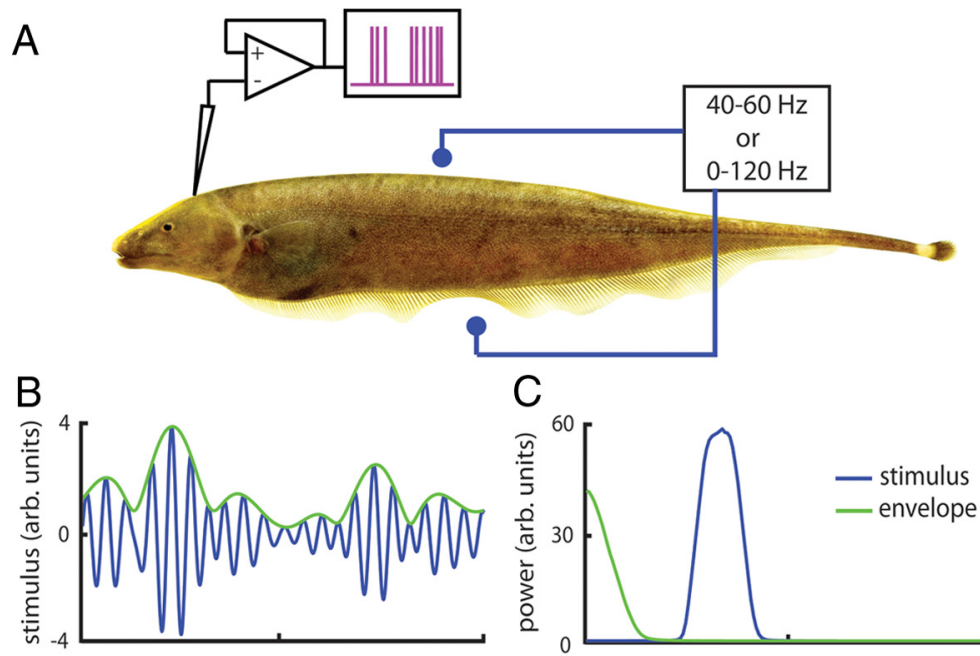

C
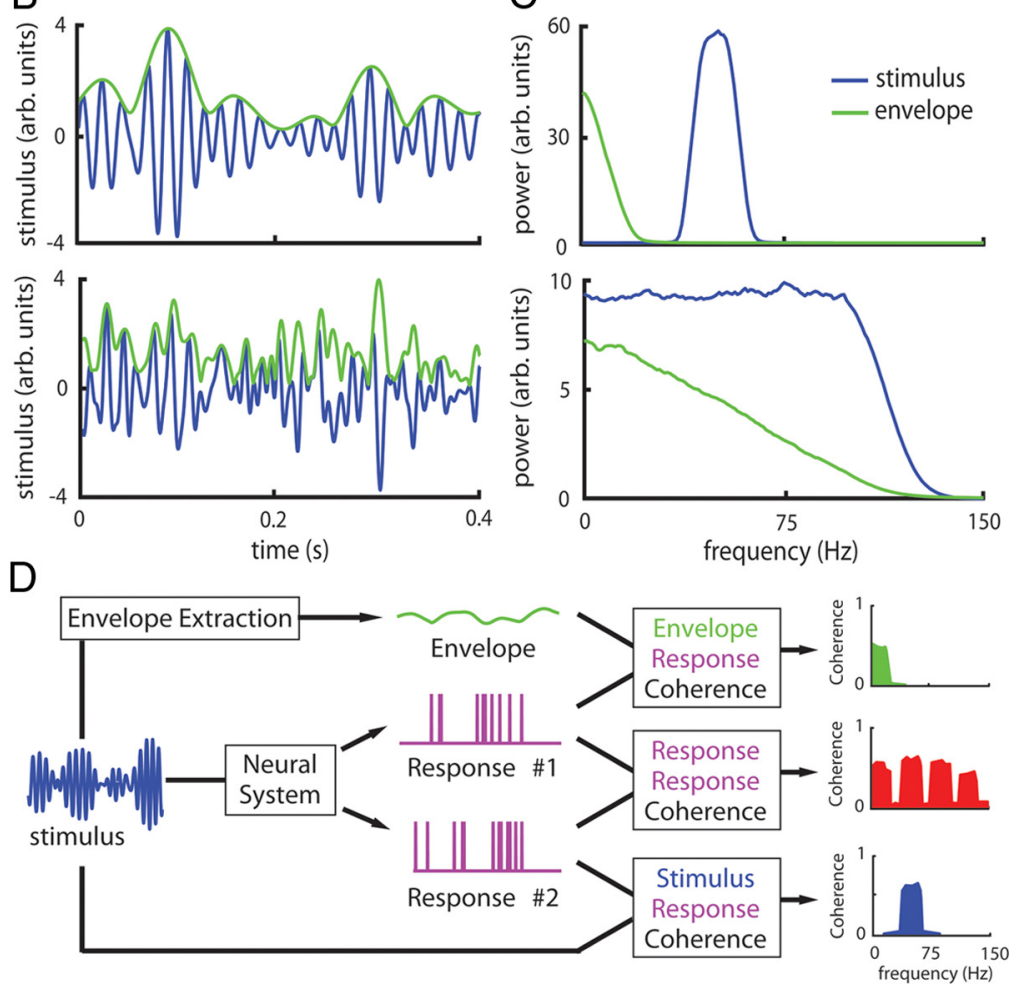

Figure 1. A, Schematic depicting the experimental paradigm. The animal was stimulated with either broadband $0-120 \mathrm{~Hz}$ or narrowband $40-60 \mathrm{~Hz}$ noise stimuli, while recordings were made from both ELL neurons and their postsynaptic target neurons within the TS. $\boldsymbol{B}$, Top, Segment of the $40-60 \mathrm{~Hz}$ narrowband noise (blue) and its low-frequency envelope (green). Bottom, Segment of the $0-120 \mathrm{~Hz}$ narrowband noise (blue) and its low-frequency envelope (green). C, Top, Power spectra of the $40-60 \mathrm{Hznoise}$ (blue) and its low-frequency envelope (green). The stimulus power spectrum contains power between $40 \mathrm{and} 60 \mathrm{~Hz}$, while the envelope contains power between 0 and $20 \mathrm{~Hz}$. Bottom, Power spectra of the $0-120 \mathrm{~Hz}$ noise (blue) and its low-frequency envelope (green). Both the stimulus waveform and its envelope contain power at frequencies between 0 and $120 \mathrm{~Hz}$. D, Summary of the methodologies used. The response to the stimulus waveform is quantified as the stimulus-response coherence. The envelope is obtained from the stimulus waveform via the Hilbert transform and the response to the envelope is quantified as the envelope-response coherence. Finally, we computed the coherence between the responses to repeated presentations of the same stimulus, the response-response coherence, which gives an upper bound on the amount of information that can be transmitted by the neuron. Note that the response-response coherence can be non-zero for frequencies outside of those contained in the stimulus waveform itself.

pe-response coherences by the response-response coherence for each neuron to quantify the response to first- and second-order attributes, respectively.

\section{Segregation of responses to first- and second-order attributes in TS neurons}

We found 20 TS neurons that responded to both first- and second-order attributes (Fig. $2 \mathrm{~A}$, first and second order). Of these neurons, 15 were excited by increases in the stimulus waveform (E-type) while the remaining 5 were inhibited (I-type). Eight TS neurons responded to first- but not to second-order attributes (Fig. 2A, first order only). These neurons were all E-type. Finally, we found 7 TS neurons that responded to second- but not to first-order attributes (Fig. $2 \mathrm{~A}$, second order only). As these neurons did not respond to the stimulus waveform itself, they cannot be segregated into E or I type. All other TS neurons did not respond to the stimuli used here.

These three different response profiles were seen using both narrowband 40-60 $\mathrm{Hz}$ and broadband noise $0-120 \mathrm{~Hz}$ input. This is important because for the $40-60$ $\mathrm{Hz}$ noise input, the frequencies contained in the stimulus waveform and its envelope are nonoverlapping, whereas for the $0-120$ $\mathrm{Hz}$ noise input, they overlap almost completely (Fig. 1C). The first order-only and second order-only neurons responded almost exclusively to the stimulus waveform and its envelope, respectively, even when they had almost complete frequency overlap with one another such as for the broadband noise stimulus. Therefore the differences between the responses of first order-only and second order-only neurons are not due to some simple form of frequency filtering.

Further, these three groups of neurons can be distinguished based on their responses to first- and second-order stimulus attributes (Fig. 2B): three distinct clusters are seen in a plot of the normalized secondorder response as a function of the normalized first-order response. The neurons that responded to both first and second order formed a cluster near the identity line, indicating similar response magnitude to both. In contrast, second order-only neurons formed a cluster above the identity line while first order-only neurons formed a cluster below the identity line. The distributions of normalized first- and second-order responses were both bimodal $(p=0.001$ and 0.002 , respectively, Hartigan's dip test). These results strongly suggest that these three neuron groups correspond to distinct subpopulations within the TS.

\section{ELL neurons respond to both first- and second-order attributes}

Perhaps the simplest explanation of our observed TS neuronal responses is that they are inherited from their afferent ELL pyramidal neurons. Interestingly, there are two types of pyramidal cells: E-type pyramidal cells receive direct synaptic excitation from afferents and respond to increases in EOD amplitude whereas I-type pyramidal cells receive synaptic inhibition from local interneurons and thus respond to decreases in EOD amplitude (Maler, 1979, 1981; Saunders and Bastian, 1984). E- and I-type pyramidal cells correspond to the ON- and OFF-type cells found in other systems, respectively.

We therefore characterized the responses of ELL neurons to the same $40-60 \mathrm{~Hz}$ narrowband and $0-120 \mathrm{~Hz}$ broadband noise inputs. All ELL neurons responded to both first- and secondorder attributes of these stimuli. Further, our results show that 
both E-type $(N=29)$ and I-type $(N=37)$ ELL neurons responded to both the stimulus and the envelope (Fig. 3A). Plotting the normalized second-order response as a function of the normalized first-order response for ELL neurons gave rise to one cluster of points that was located below the identity line (Fig. 3B). The distributions of normalized first- and secondorder responses were both unimodal $(p=$ 0.95 and 0.79 , Hartigan's dip test, respectively). Finally, E- and I-type ELL pyramidal neurons did not differ significantly in their tendencies to respond to either firstor second-order stimulus attributes, respectively (Fig. $3 C, D$ ), and thus were pooled in subsequent analysis.

Previous studies have shown that ELL neurons display large heterogeneities in their responses to sensory input that are correlated with their baseline firing rates (Bastian et al., 2002, 2004; Chacron et al., 2005c, 2007; Chacron, 2006; Toporikova and Chacron, 2009; Avila-Akerberg et al., 2010). Therefore, we next looked at whether ELL pyramidal neurons might respond differentially to first- and second-order attributes depending on their baseline firing rates. We plotted the normalized first-order response as a function of the baseline firing rate (Fig. $4 A$ ) and found a significant positive correlation $(r=0.57, p<0.01, N=66)$. In contrast, we found a negative correlation between the normalized second-order response and the baseline firing rate (Fig. $4 B)(r=-0.40, p<0.01, N=66)$. These data show that ELL neurons with the highest firing rates tend to respond preferentially to first-order attributes while ELL neurons with the lowest firing rates tend to respond preferentially to second-order attributes. Thus, one possible explanation for the presence of first order-only neurons in TS is that they receive input preferentially from ELL pyramidal neurons with high firing rates. We shall return to this point below.

What causes ELL neurons to respond preferentially to either first- or second-order stimulus attributes? Examination of ELL neuron responses revealed that those with the lower firing rates tended to be reliably driven into cessation of firing for some portions of the stimulus (i.e., rectification) (Fig. 4C). Such rectification is accompanied by phase locking since the onset of action potential firing must then occur reliably at other portions of the stimulus (Savard et al., 2011; Schneider et al., 2011). In contrast, ELL neurons with higher firing rates tended to fire action potentials throughout the stimulus and, as such, tended to display less phase locking (Fig. 4D). We then quantified phase locking in ELL pyramidal neurons as a function of their baseline firing rates and found a significant negative correlation between both quantities $\left(r=-0.66, p \ll 10^{-3}, N=66\right)$ (Fig. 4E). Together with our result that the normalized second-order response was negatively correlated with the baseline firing rate, this shows that it is the pyramidal cells with the lowest baseline firing rates that tend to display the most phase locking as well as the strongest secondorder responses.

\section{Balanced input from E- and I-type sources determines envelope selectivity}

What makes TS neurons respond selectively to second-order attributes? An important clue came from STC analysis. In contrast to the STA, which gives the average stimulus feature that triggers an action potential, STC analysis focuses on the variability around the STA. The idea is that the structure of this variability can reveal whether a neuron receives input from multiple sources (Agüera y Arcas et al., 2003; Slee et al., 2005; Fairhall et al., 2006; Gollisch and Meister, 2008).

STC analysis revealed that the TS neurons that responded to both first- and second-order attributes displayed little variability around the STA (Fig. 5A). Plotting the covariance matrix revealed that the variability around the STA was less than that expected from the stimulus alone just before the spike, implying that stimulus features resembling the STA reliably elicit spiking activity (Fig. 5C). In contrast, neurons that responded selectively to second-order attributes displayed significant variability around the STA (Fig. 5B), and the covariance matrix revealed a large increase in variability that was greater than that expected from 
A

E-type (ON)
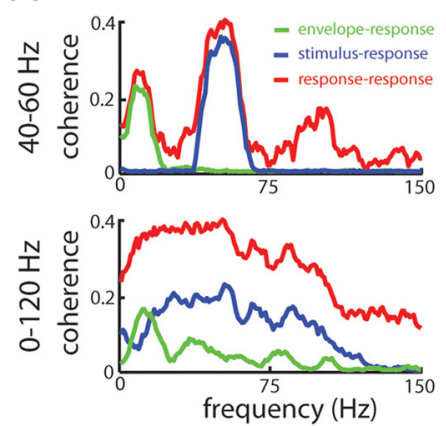

B

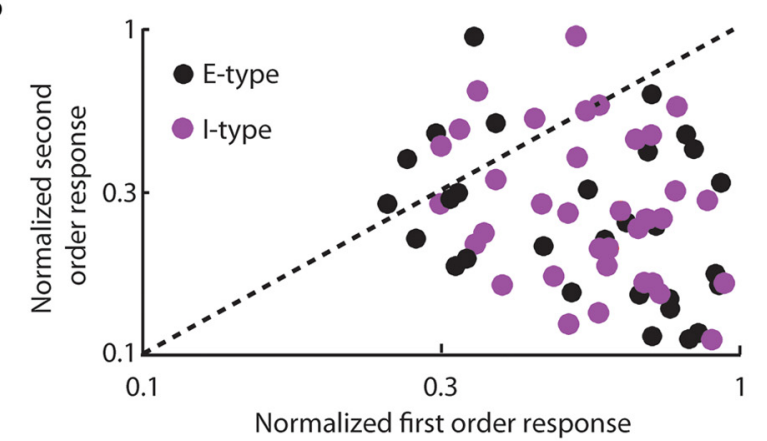

C

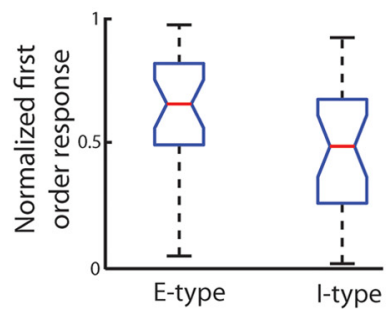

I-type (OFF)
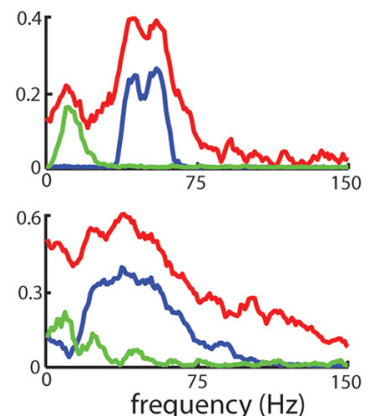

50

equal contributions. E-type TS neurons that responded to both first- and second-order attributes displayed bias indices near 1, while I-type TS neurons that responded to both first- and second-order attributes displayed bias indices near -1 (Fig. 5G). In contrast, TS neurons that responded only to second-order attributes displayed bias indices near 0 (Fig. $5 G$ ).

We note that no significant differences in firing rates were observed between the three groups of TS neurons ( $p>0.1$, oneway ANOVAs). Further, E- and I-type TS neurons did not differ significantly in their firing rates or responses to first- and secondorder stimulus attributes $(p>0.1$ in all cases, Wilcoxon rank sum tests). Finally, no significant correlations were observed for TS neurons between firing rate and either of normalized first $(r=$ $0.34, p=0.068, N=35)$ - or second $(r=0.09, p=0.64, N=$ $35)$-order response. Together, these results are consistent with the hypothesis that TS neurons combine E- and I-type inputs differentially to respond selectively to either first- or secondorder stimulus attributes.

\section{Modeling convergent ELL inputs onto TS neurons}

To further explore the hypothesis that the relative balance between E- and I-type inputs onto TS neurons determines whether a neuron can respond selectively to second-order attributes, we built a model in which we could vary the relative strengths of inputs from E- and I-type ELL pyramidal cells onto a TS neuron (Fig. 6A). We systematically varied the fraction of E-type input $\rho_{\mathrm{E}}$ received by our model TS neuron: when $\rho_{\mathrm{E}}=0$, the model TS neuron receives input only from the I-type ELL pyramidal neuron while, when $\rho_{\mathrm{E}}=1$, the model TS neuron receives input only from the E-type ELL pyramidal neuron. The case where both inputs are balanced in strength thus corresponds to $\rho_{\mathrm{E}}=0.5$.

This relatively simple model does a good job of reproducing the responses of TS neurons to both $40-60 \mathrm{~Hz}$ and $0-120 \mathrm{~Hz}$ noise stimuli. For $\rho_{\mathrm{E}}=0.9$ and $\rho_{\mathrm{E}}=0.1$, the model reproduces the responses of E- and I-type first- and second-order TS neurons, respectively (compare Figs. $6 B$ and $2 A$ ). Moreover, when $\rho_{\mathrm{E}}=0.5$, our model TS neuron displayed a strong response to second-order attributes and almost no response to first-order attributes, which is comparable to data from second order-only TS neurons (compare Figs. $6 B$ and $2 A$ ).

We applied the STC analysis to our model TS neuron in the same manner as for the experimental data. For $\rho_{\mathrm{E}}=0.9$ and $\rho_{\mathrm{E}}=0.1$, the spike-triggered covariance matrices displayed significantly less variance than that expected from the stimulus just before the spike and resembled that obtained from TS neurons that responded to both first- and second-order attributes (compare Figs. $6 C$ and $5 C$ ). In contrast, for $\rho_{\mathrm{E}}=0.5$, the spike-triggered covariance matrix displayed greater variance than that expected from the stimulus just before the spike and resembled that obtained from second orderonly TS neurons (compare Figs. $6 C$ and $5 D$ ). We also computed the E and I filters from our model neuron. When $\rho_{\mathrm{E}}=0.1$, the I filter was much stronger in magnitude than the $\mathrm{E}$ filter (Fig. $6 D$ ). In contrast, when $\rho_{\mathrm{E}}=0.9$, the E filter was much stronger in magnitude than the I filter (Fig. 6D). Moreover, the $\mathrm{E}$ and I filter obtained resembled those of neurons that responded to both first- and second-order attributes (compare Figs. $6 D$ and $5 E$ ). When $\rho_{\mathrm{E}}=0.5$, the E and I filters were opposite in sign and were approximately equal in mag- 
A

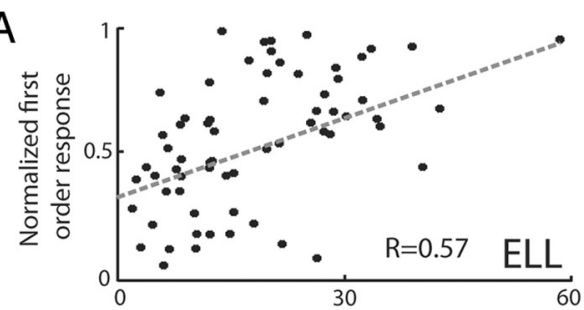

B

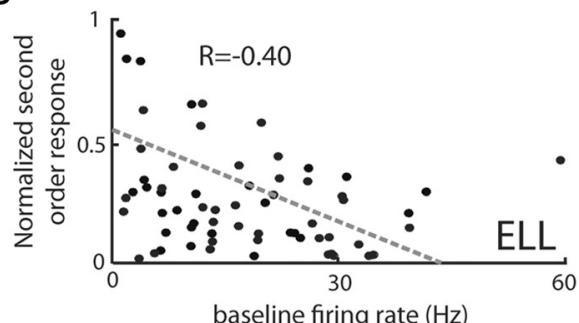

C

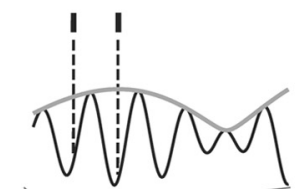

\begin{tabular}{|l|lll}
\hline II & I & III I I
\end{tabular}

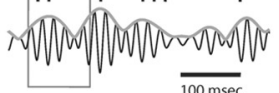

D $\quad 34 \mathrm{~Hz}$
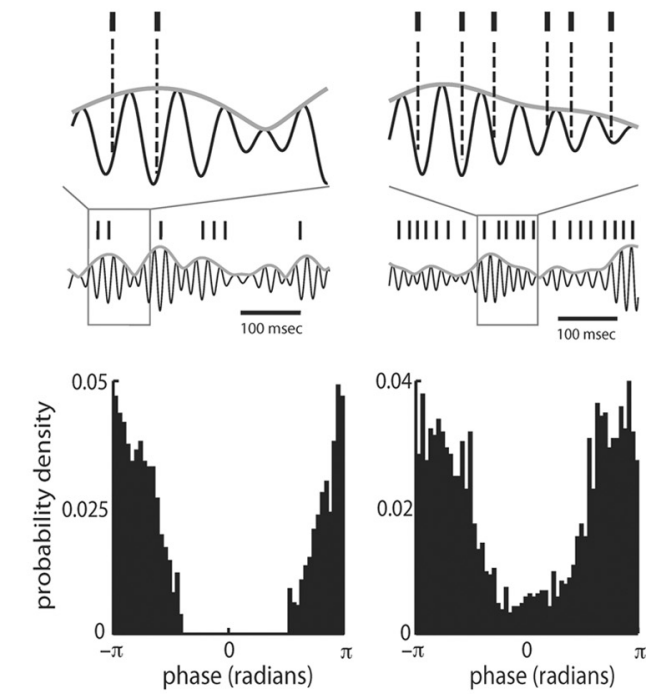

$E$

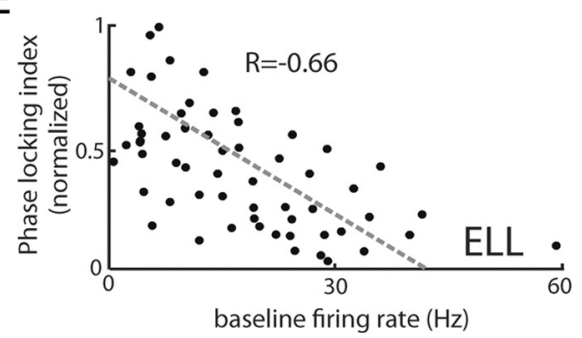

Figure 4. Heterogeneities among ELL neurons influence first- and second-order coding. $A$, Normalized first-order response as a function of baseline firing rate for ELL neurons. A significant positive correlation was observed $\left(r=0.53, p<10^{-3}, N=48\right)$. $B$, Normalized secondorder response as a function of baseline firing rate for ELL neurons. A significant negative correlation was observed $(r=-0.35, p=0.01, N=48)$. C, Response of an example l-type ELL neuron with low firing rate $(16 \mathrm{~Hz})$ to the $40-60 \mathrm{~Hz}$ stimulus waveform (black) and its envelope (gray). The vertical bars indicate the spike times. This neuron exhibited phase locking to the stimulus as the spikes occurred preferentially near a local minimum of the stimulus waveform. The phase histogram (bottom) shows that this neuron was rectifying as the probability of firing was zero for phases lesser than $\pi / 2$ in magnitude. This neuron also tended to fire action potential when the envelope was higher. Similar results were seen for E-type ELL neurons with low firing rates except that these tended to fire action potentials near a local maximum of the stimulus (data not shown). $\boldsymbol{D}$, Response of an example l-type ELL neuron with high firing rate ( $34 \mathrm{~Hz}$ ) to the $40-60 \mathrm{~Hz}$ stimulus waveform (black) and its envelope (gray). The vertical bars indicate the spike times. Although the action potentials occurred near a local minimum, this neuron displayed less phase locking and did not display as much rectification as seen from its
A first and second order

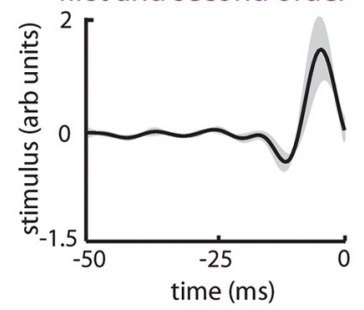

B second order only
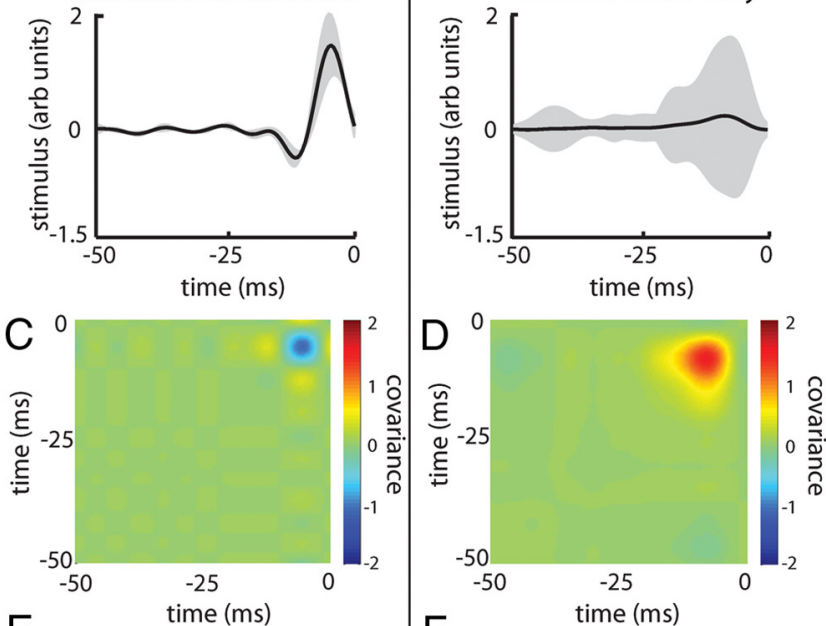

$\mathrm{E}$
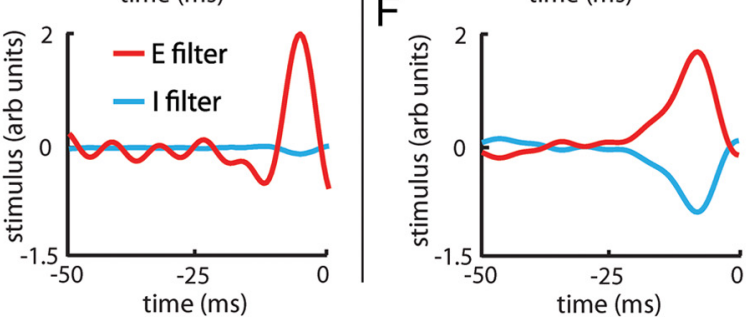

G

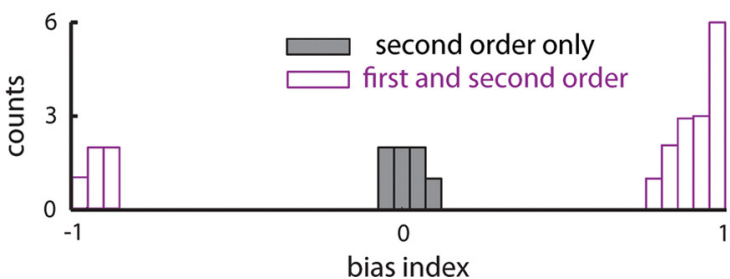

Figure 5. The relative proportion of E-and I-type inputs unto TS neurons determines their secondorder response selectivity. A, STA (black) from a typical first- and second-order TS neuron. The gray band shows the SD around the STA that was relatively small for this neuron. $\boldsymbol{B}$, Spike-triggered average (black) from a typical second order only TS neuron. The gray band shows the SD around the STA that was, in contrast, quite large. C, The covariance matrix with prior subtracted from the same firstand second-order TS neuron shows a large negative value just before the spike at time 0 indicating that stimulus features that resemble the STA reliably elicit spiking in this neuron. $\boldsymbol{D}$, The covariance matrix with prior subtracted from the same second order only TS neuron shows a large positive value just before the spike at time 0 indicating that multiple stimulus features that differ significantly from the STA elicit spiking in this neuron. E, Eand I filters from the same first-and second-order TS neuron. The E filter (red) was large and resembled the STA in shape while the I filter (blue) was close to $0 . \boldsymbol{F}$, E and I filters from the same second order only TS neuron were opposite in sign and matched in magnitude. $\mathbf{G}$, The bias index, measuring the relative proportion of l-input to E-input for a given neuron in $T S$, is near 1 for E-type neurons that respond to first-and second-order stimulus features, near -1 for I-type neurons that respond to first- and second-order stimulus features, and near 0 for second order only neurons.

nitude, which is similar to what is observed for second order-only neurons (compare Figs. $6 D$ and $5 F$ ).

We next systematically varied the parameter $\rho_{\mathrm{E}}$ in our model and found that the envelope-response was maximal and the stimulus-response was minimal for $\rho_{\mathrm{E}}=0.5$ (Fig. 7A). Moreover, the bias index had a sigmoidal dependency on $\rho_{\mathrm{E}}$ (Fig. $7 B$ ), which might explain the strong magnitude of the bias index observed

phase histogram (bottom). Note that similar responses were observed for E-type pyramidal neuron except that these tended to respond preferentially near a local maximum of the waveform (data not shown). $\boldsymbol{E}$, Phase locking index as a function of baseline firing rate for ELL neurons. Both quantities were significantly negatively correlated $\left(r=-0.66, p<10^{-3}, N=66\right)$. 
A

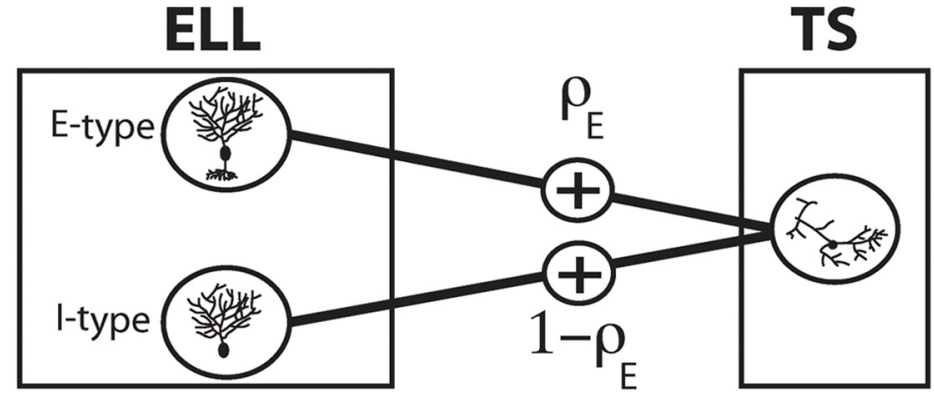

B

$\rho_{E}=0.1$

$\rho_{E}=0.5$

$\rho_{E}=0.9$
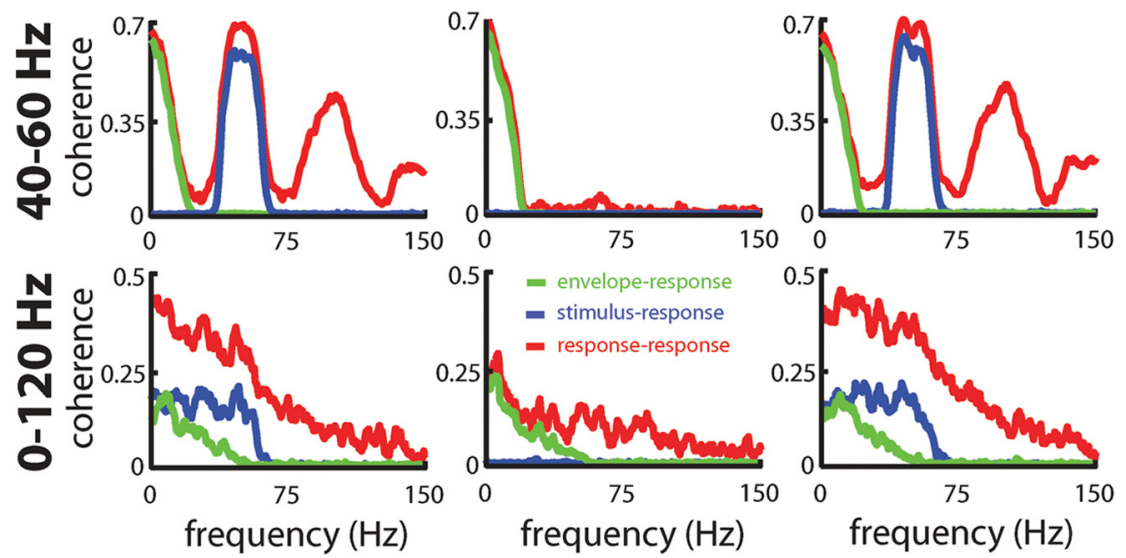

C
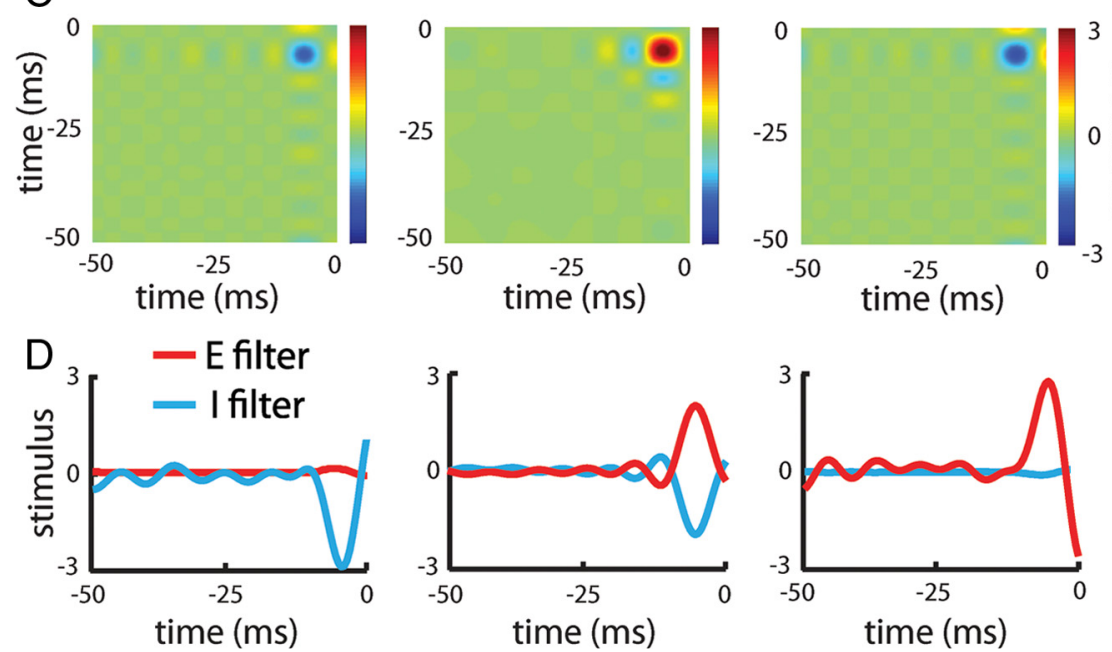

Figure 6. Modeling convergent excitatory E- and I-type input from ELL onto TS neurons. $A$, Model schematic showing inputs from E-and I-type ELL neurons exciting our model TS neuron. The parameter $\rho_{\mathrm{E}}$ is the fraction of E-type input received by the model TS neuron. $B$, Responses as quantified by the coherence function of our model TS neuron to $40-60 \mathrm{~Hz}$ (top) and $0-120 \mathrm{~Hz}$ (bottom) noise stimulation for $\rho_{\mathrm{E}}=0.1$ (left), $\rho_{\mathrm{E}}=0.5$ (center), and $\rho_{\mathrm{E}}=0.9$ (right). C, Covariance matrices with prior subtracted from our model TS neuron for $\rho_{\mathrm{E}}=0.1$ (left), $\rho_{\mathrm{E}}=0.5$ (center), and $\rho_{\mathrm{E}}=0.9$ (right). $\boldsymbol{D}, \mathrm{E}$ (blue) and I (red) filters from our model TS neuron for $\rho_{\mathrm{E}}=0.1$ (left), $\rho_{\mathrm{E}}=0.5$ (center), and $\rho_{\mathrm{E}}=0.9$ (right).

for E- and I-type neurons that responded to both first- and second-order attributes (Fig. 5G). This sigmoidal dependency results from the fact that the output bias depends solely on the spiking, which is a nonlinear mechanism, whereas the input bias does not. As such, the output bias does not take into account subthreshold membrane potential fluctuations that do not give rise to spiking and can thus be greater in magnitude than the input bias.
Finally, we return to the hypothesis that first order-only TS neurons receive input exclusively from ELL pyramidal neurons with the highest firing rates. Since all first order-only TS neurons were E-type, we assumed that they received input only from E-type ELL pyramidal neurons and set $\rho_{\mathrm{E}}=1$ in our model. We next systematically varied the baseline firing rate of the input E-type ELL pyramidal neuron over the physiological range. Increasing the baseline firing rate of the ELL model neuron tended to decrease its response to second-order attributes and increase its response to first-order attributes (Fig. 7C), which is consistent with experimental data (compare Figs. $7 C$ and $4 A, B)$. Moreover, we found that such a manipulation did also lead to an increased firstorder and decreased second-order response in the model TS neuron (Fig. 7D).

\section{Verifying the model's prediction}

If second order-only TS neurons receive balanced excitatory input from E- and I-type ELL neurons, then we would expect that these neurons would respond in a biphasic manner to sinusoidal stimuli. This is because E- and I-type ELL neurons respond to such stimuli with spike trains that are out of phase with respect to one another (Bastian et al., 2002).

Thus, we next recorded the responses of TS neurons to $4 \mathrm{~Hz}$ sinusoidal stimuli. First- and second-order TS neurons responded to such stimuli by firing near a preferred phase of the input (Fig. $8 \mathrm{~A}$ ) as confirmed by a phase histogram that was unimodal (Fig. $8 \mathrm{~B}$ ). In contrast, second order-only TS neurons instead responded by firing near two distinct phases of the stimuli that were separated by $\pi$ radians (Fig. $6 C$ ), and had a bimodal phase histogram (Fig. 8D). We further found a strong positive correlation between the bimodality and second-order response selectivity as the first- and second-order and second order-only TS neurons formed distinct clusters $\left(r=0.94, p \ll 10^{-3}, N=20\right)$ (Fig. $8 E$ ). Indeed, both the distributions of bimodality and second-order response selectivity were significantly bimodal (Hartigan's dip test, $p<10^{-3}$ in

both cases, $N=20$ ). Further, a strong and significant correlation between bimodality and second-order response selectivity was also observed when we only considered second order-only TS neurons $(r=0.78, p=0.04, N=7)$. These results confirm our model's prediction that the relative balance between the $\mathrm{E}$ and I inputs unto TS neurons determine their response selectivity to second-order stimulus attributes. 

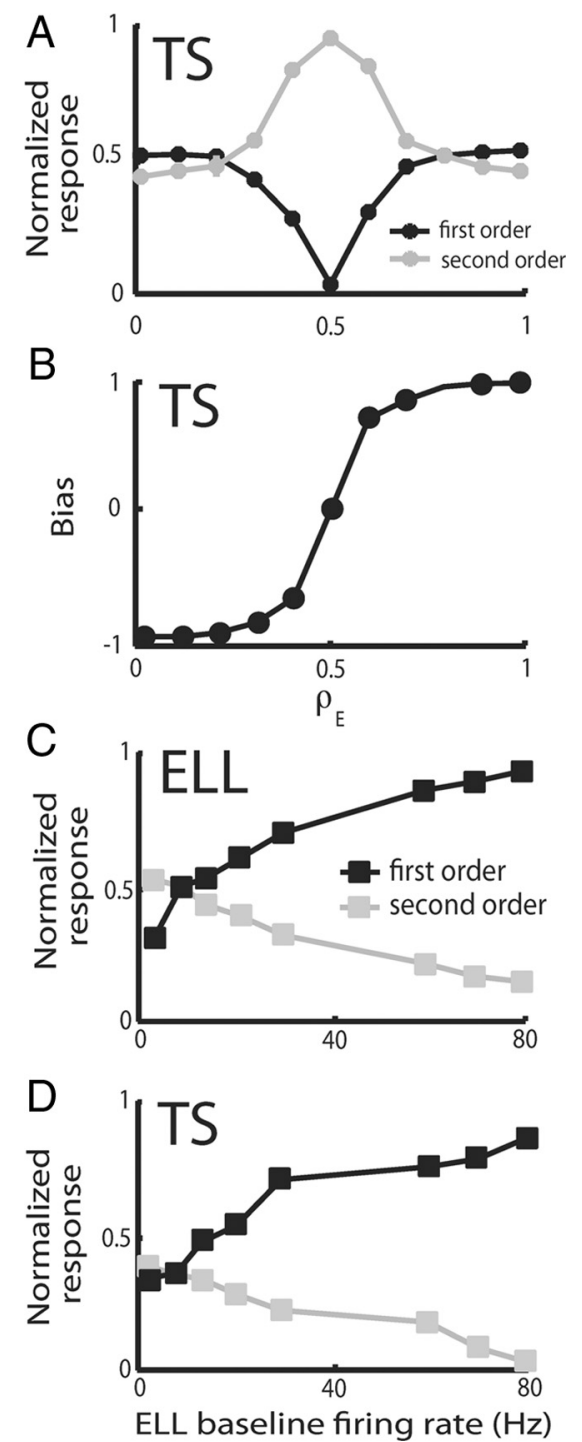

Figure 7. Explaining the emergence of selectivity to first- and second-order stimulus attributes in TS neurons. $\boldsymbol{A}$, Normalized first-order (black) and second-order (gray) responses of our model TS neuron as a function of $\rho_{\mathrm{E} \cdot}$, B, Bias index as a function of $\rho_{\mathrm{E}^{\mathrm{E}}}$. C, Normalized first-order (black) and second-order (gray) responses of our model ELL neuron as a function of the ELL baseline firing rate. $\boldsymbol{D}$, First-order (black) and second-order (gray) responses of our model TS neuron as a function of the ELL baseline firing rate.

Understanding the nature of the neural circuits by which stimulus and envelope selectivity emerges in TS

How does combining E- and I-type inputs in a balanced fashion lead to a response that is selective to the second-order attributes as quantified by the envelope? Our results show that both E- and I-type ELL neurons that respond to second-order attributes also tend to display rectification: the responses of E- and I-type neurons are illustrated in Figure 9A. Both neurons are phase locked to the stimulus waveform and tend to fire at different phases: E-type neurons tend to fire near a local maximum, while I-type neurons tend to fire near a local minimum. As such, the responses of each neuron tend to be $180^{\circ}$ out of phase with respect to the fine structure of the stimulus waveform (i.e., first-order attributes). The situation is different in relation to the envelope as both E- and I-type pyramidal neurons tend to fire more action potentials when the envelope increases (Fig. 9A). Both responses are more in phase with one another with respect to the envelope.
A first and second order C second order only
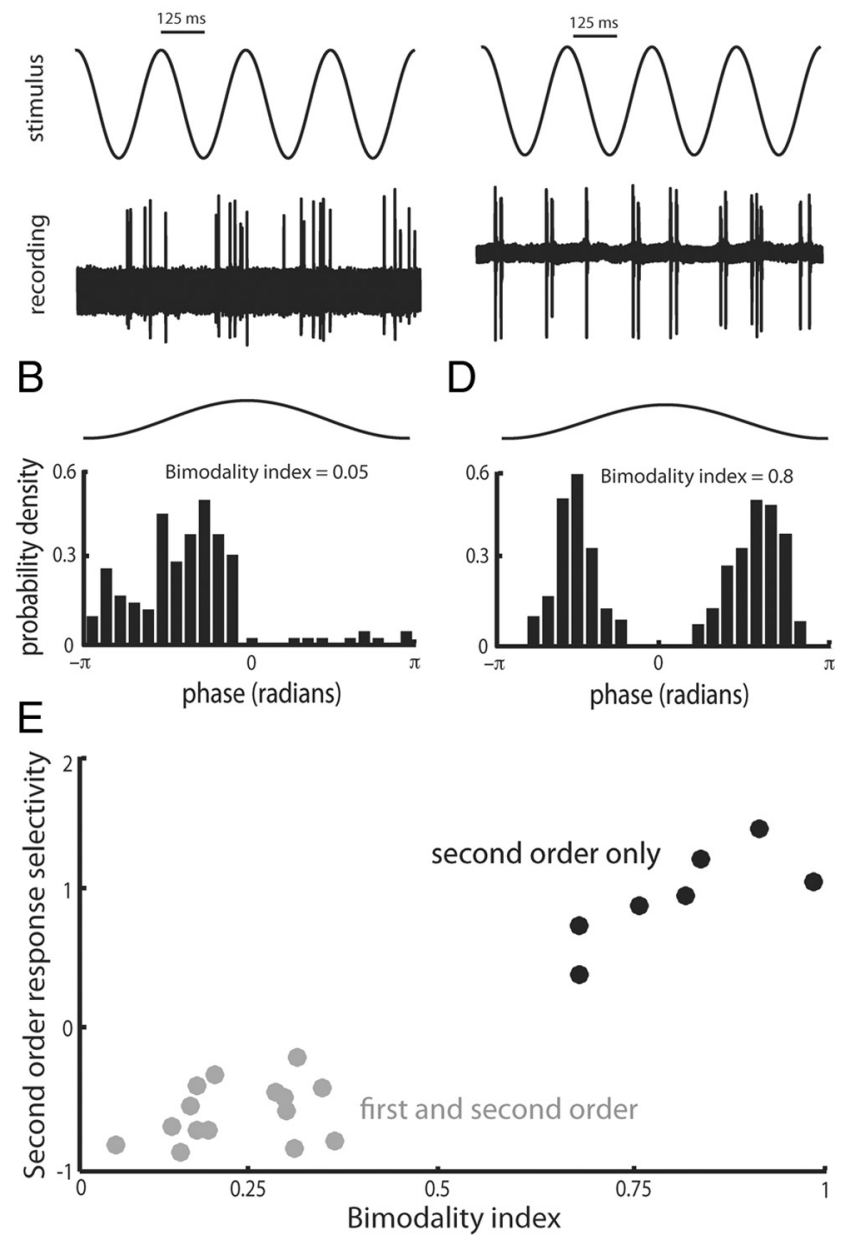

phase (radians)

Figure 8. Testing the model's prediction that second order-only TS neurons receive balanced input from both E- and I-type sources. A, Response (bottom) of an example first- and secondorder TS neuron to $4 \mathrm{~Hz}$ sinusoidal stimulation (top). Spikes tend to occur on the rising phase of the stimulus. $\boldsymbol{B}$, Unimodal phase histogram from this same neuron confirming that spikes preferentially occur on the rising phase of the stimulus. One cycle of the stimulus is shown on top. C, Response (bottom) of an example second order-only TS neuron to $4 \mathrm{~Hz}$ sinusoidal stimulation (top). This neuron responds preferentially at two phases of the input. $\boldsymbol{D}$, Bimodal phase histogram from this same neuron confirming that spikes preferentially occur at two different phases of the stimulus that are separated by $\pi$ radians. One cycle of the stimulus is shown on top. $E$, Second-order response selectivity as a function of bimodality index for second order-only TS neurons (black dots) as well as first- and second-order TS neurons (gray dots). A significant positive correlation is observed between both quantities when all the data are considered $(r=$ $\left.0.94, p \ll 10^{-3}, N=20\right)$ as well as when second order-only TS neurons were considered exclusively $(r=0.78, p=0.04, N=7)$.

(i.e., second-order attributes). Adding the spike trains of E- and I-type neurons results in a response that is not correlated with first-order stimulus attributes because of destructive interference, but is correlated with second-order attributes because of constructive interference.

Together, our results suggest that: (1) E-type ELL pyramidal neurons with the highest baseline firing rates project preferentially to first order-only TS neurons, (2) second order-only TS neurons receive balanced excitatory input from both E- and I-type ELL neurons, and (3) TS neurons that respond to both first- and second-order attributes receive excitatory input from both E- and I-type ELL neurons but in an unbalanced fashion. This proposed circuit is shown in Figure $9 B$ and is further discussed below. 
A second order only II IIIIIII IIIIII IIII IIII
TS neuron
envelope
ELL neuron
stimulus
I-type
ELL neuron

B

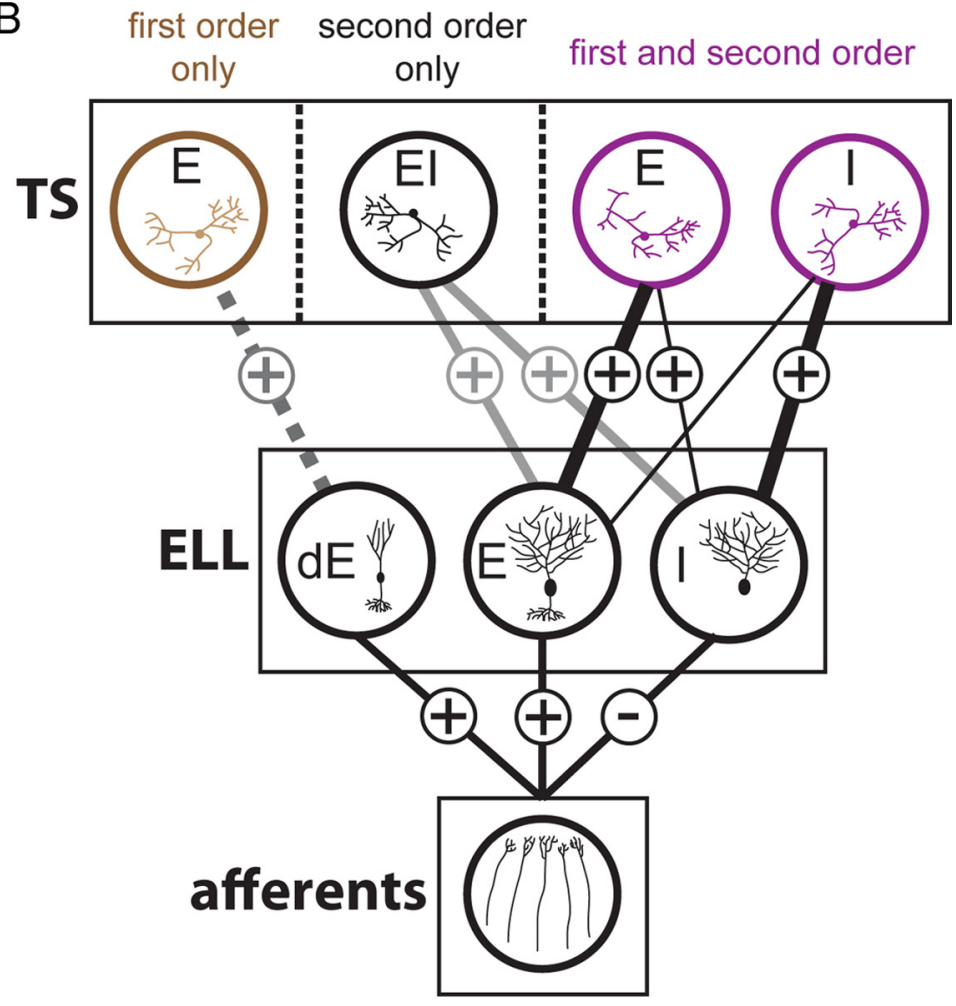

Figure 9. Summary of the proposed neural circuit that mediates parallel coding of first- and second-order stimulus attributes in TS. A, Example spike trains (vertical bars) with the stimulus waveform of E-type (blue) and I-type (red) ELL neurons. E-type neurons respond near local maxima of the stimulus waveform but their probability of firing increases with the envelope (black). In contrast, I-type neurons respond near local minima of the stimulus waveform but their probability of firing also increases with the envelope. Adding their spike trains (black vertical bars) results in a response that is correlated with the envelope (second order) but not the stimulus waveform (first order). B. Electrosensory afferents excite E-type and inhibit l-type ELL pyramidal neurons. However, E-type pyramidal neurons with the highest firing rates (dE) tend to respond more to first-order attributes and less to second-order attributes and are assumed to excite first order-only TS neurons since these neurons were all found to be E-type. E- and I-type firstand second-order TS neurons receive input from both $\mathrm{E}$ - and I-type pyramidal cells but in an unbalanced fashion such that the proportion of E-type and I-type ELL input is greatest for E- and I-type first- and second-order TS neurons, respectively. In contrast, both sources of ELL input are matched in strength for second-order-only TS neurons.

\section{Discussion}

\section{Summary of results}

We recorded the responses of TS and ELL neurons to narrowband $(40-60 \mathrm{~Hz})$ and broadband $(0-120 \mathrm{~Hz})$ noise stimuli and investigated their responses to first- and second-order stimulus attributes. We found three distinct TS neuron groups: one responded both to first- and second-order attributes, one responded only to first-order attributes, and one responded only to second-order attributes. In contrast, afferent ELL neurons always responded to both first- and second-order attributes. However, ELL neurons with the highest baseline firing rates tended to respond the most to first-order attributes and the least to second- order attributes because they displayed the least phase locking. A combination of spike-triggered covariance analysis and mathematical modeling revealed that the relative balance between the E- and I-type inputs received by TS neurons determines whether they respond to both first- and second-order attributes, first-order attributes only, or second-order attributes only. Interestingly, when both E- and I-type inputs to a model TS neuron were matched in strength, the neuronal spiking activity closely resembled that seen experimentally in second order-only TS neurons. We then varied the firing rate of our model ELL neurons and showed that this could make our model TS neuron respond more selectively to first-order attributes as observed experimentally in first order-only TS neurons. Finally, we confirmed our model's prediction that second order-only TS neurons receive balanced input from E and I sources by recording their responses to sinusoidal stimuli and showing that they display a biphasic response.

Processing of second-order stimulus attributes in the electrosensory system Recent field studies show that electrosensory stimuli that are encountered by weakly electric fish in the wild are characterized by time-varying second-order attributes as quantified by the envelope (Stamper et al., 2010). These results were obtained subsequent to electrophysiological studies showing that electrosensory neurons respond to these envelopes (Middleton et al., 2006; Savard et al., 2011). Thus, our results showing that TS neurons can respond to second-order attributes add to a growing body of literature suggesting that these stimuli are behaviorally relevant and are processed by electrosensory neurons. In particular, we have described a category of TS neurons that selectively extracts second-order information while another category selectively extracts first-order information. Our results thus give rise to the interesting hypothesis that parallel processing of first- and second-order attributes begins at the level of the TS and is further refined in higher-order brain areas.

This parallel processing of the first- and second-order attributes of electrosensory input is likely to be of behavioral importance. Indeed, when two fish engage in agonistic or courtship behavior, first-order attributes will provide information as to the frequency difference between the two fish while second-order attributes instead gives information about the distance between and perhaps the orientation of the two fish. There is no reason to expect a correlation between both signals and previous studies have found a significant overlap between the frequency ranges of beats and envelopes (beat frequency range: $0-400 \mathrm{~Hz}$; envelope 
frequency range: $0-50 \mathrm{~Hz}$ ) (Stamper et al., 2010). We note that the first- and second-order attributes of the noise stimuli used in this study both had frequency content within the range of those that are experienced in the natural environment. Therefore, our results suggest that weakly electric fish could distinguish between first- and second-order attributes of such sensory input even when they overlap in their frequency content by using first and second order-only TS neurons, respectively.

The TS neurons that responded selectively to second-order attributes received balanced input from E- and I-type pathways. As such, they most likely correspond to the previously described E-I neurons that are excited by both increases and decreases in the stimulus (Partridge et al., 1981; Rose and Call, 1993). The most parsimonious explanation for their properties is that they receive balanced excitation from E- and I-type ELL neurons. Of course, it is possible that such inputs could come from E- and I-type neurons within the TS itself. This scenario is possible but unlikely because I-type neurons are relatively rare within the TS. Another possibility is that E-I neurons would receive synaptic excitation from E-type TS neurons and synaptic inhibition from another group of E-type TS neurons. This scenario is also possible but unlikely because a delay would then have to be introduced for the inhibition, which is inconsistent with our experimental observations that the E- and I-type inputs were not significantly delayed with respect to one another.

Our results showed that the responses of ELL pyramidal neurons to second-order attributes and their tendency to display phase locking are both negatively correlated with their baseline firing rates. Phase locking in ELL pyramidal cells is most likely due in part to the amount of inhibition that they receive from interneurons (Middleton et al., 2006). Further, while all pyramidal cells project to TS, there is evidence that pyramidal cells with the highest firing rates constitute a distinct class as they project exclusively to some areas (Bastian and Courtright, 1991; Bastian et al., 2004). Thus, our results suggest that it is these pyramidal cells with the highest firing rates that project preferentially to stimulus-only TS neurons. Further, they suggest that pyramidal cells with the lowest firing rates would tend to project to second order-only and first- and second-order TS neurons as they tended to show strong responses to both first- and second-order stimulus attributes. As such, our results provide new insight as to how different pyramidal cell classes have different functional roles.

All three neuron types that were found in TS formed distinct clusters when we plotted their second-order responses as a function of their first-order responses (Fig. 2B), suggesting that they correspond to specific groups of cells within TS. While the anatomy of the different cell classes within TS have been thoroughly characterized (Carr and Maler, 1985), the only correlations between anatomy and physiology that have been investigated to date are between position in the different layers of the TS and between the density of spines (Rose and Call, 1992, 1993; Fortune and Rose, 1997a,b). Further studies using intracellular recordings and quantitative analysis of neuronal morphologies are needed to verify our data and are beyond the scope of this paper.

Finally, our model shows that integration of afferent ELL input by TS neurons is sufficient to give rise to parallel processing of first- and second-order stimulus attributes. This is surprising given that anatomical studies have reported significant connections between TS neurons (Carr and Maler, 1985). Based on previous studies (Mehaffey et al., 2007; George et al., 2011), it is conceivable that such connections could further refine parallel processing of first- and second-order stimulus attributes in TS neurons. Further experiments are however required to test this hypothesis.

\section{Parallel processing of first- and second-order stimulus attributes in other systems}

Our results also have important consequences for the processing of first- and second-order stimulus attributes in other systems. Indeed, time-varying second-order attributes occur in natural stimuli in the form of low-frequency envelopes and often contain behaviorally relevant information (Ahissar et al., 2000; Joris et al., 2004; Curtis and Kleinfeld, 2009). Our results suggest a general strategy for designing brain circuits that selectively extract envelope information. Specifically, we showed that neurons that receive balanced input from E- and I-type afferent neurons (i.e., E-I neurons) respond selectively to these envelopes. Neurons with response profiles similar to those of E-I neurons have previously been described in other systems. In particular, ON-OFF retinal ganglion cells receive input from both ON and OFF-type bipolar cells and respond reliably to changes in contrast (Greschner et al., 2006). In the visual system, selective lesions of the ON pathway gives rise to a large loss in contrast sensitivity (Schiller et al., 1986), which is consistent with the hypothesis that contrast sensitivity requires integration of the $\mathrm{ON}$ and OFF pathways. Moreover, type 3 neurons respond with excitation to both ipsi and contralateral rotations (Duensing and Schaefer, 1958) and have been found in several brain areas including the posterior parietal cortex (Klam and Graf, 2003). However, our spike-triggered covariance analysis revealed that E-I neurons did not respond to first-order stimulus attributes because they received balanced input from E- and I-type pathways. We suggest that these neurons might have been previously ignored because of their lack of responses to first-order stimulus attributes and that they might be found more commonly in sensory systems.

Finally, we have shown that first and second order-only neurons were not responsive to second and first-order attributes, respectively, even when the signals corresponding to both attributes strongly overlapped in their frequency contents. This strongly suggests that these neurons each encode their preferred attribute independently of the value of the other. As such, our results our provide the first experimental evidence suggesting that the CNS can remove ambiguity that is inherent in adaptive neural codes through parallel encoding of first- and second-order attributes. Further studies are however needed to test whether first and second order-only neurons actually encode first- and second-order attributes regardless of second and first-order attributes, respectively. Moreover, integration of these parallel streams of information is then required. The mechanisms that mediate such integration are currently unknown and should be the focus of subsequent investigations.

\section{Conclusions}

We have shown the first experimental evidence for parallel processing of first- and second-order stimulus attributes by electrosensory midbrain neurons. Through a combination of computational analysis and mathematical modeling, we have suggested generic neural circuitry that mediates such processing. Such circuitry will most likely be found in other systems.

\section{References}

Agüera y Arcas B, Fairhall AL (2003) What causes a neuron to spike? Neural Comput 15:1789-1807.

Agüera y Arcas B, Fairhall AL, Bialek W (2003) Computation in a single neuron: Hodgkin and Huxley revisited. Neural Comput 15:1715-1749. 
Ahissar E, Sosnik R, Haidarliu S (2000) Transformation from temporal to rate coding in a somatosensory thalamocortical pathway. Nature 406:302-306.

Attias H, Schreiner CE (1997) Low-order temporal statistics of natural sounds. Adv Neural Inf Process Syst 9:27-33.

Avila-Akerberg O, Krahe R, Chacron MJ (2010) Neural heterogeneities and stimulus properties affect burst coding in vivo. Neuroscience 168:300313.

Bair W, Movshon JA (2004) Adaptive temporal integration of motion in direction-selective neurons in macaque visual cortex. J Neurosci 24:7305-7323.

Baker CL Jr (1999) Central neural mechanisms for detecting second-order motion. Curr Opin Neurobiol 9:461-466.

Barlow HB (1961) Possible principles underlying the transformation of sensory messages. In: Sensory Communication (Rosenblith W, ed). Cambridge, MA: MIT.

Bastian J (1974) Electrosensory input to the corpus cerebelli of the highfrequency electric fish Eigenmannia virescens. J Comp Physiol 90:1-14.

Bastian J (1981) Electrolocation I. How the electroreceptors of Apteronotus albifrons code for moving objects and other electrical stimuli. J Comp Physiol A Neuroethol Sens Neural Behav Physiol 144:465-479.

Bastian J, Courtright J (1991) Morphological correlates of pyramidal cell adaptation rate in the electrosensory lateral line lobe of weakly electric fish. J Comp Physiol A Neuroethol Sens Neural Behav Physiol-Sensory Neural Behav Physiol 168:393-407.

Bastian J, Chacron MJ, Maler L (2002) Receptive field organization determines pyramidal cell stimulus-encoding capability and spatial stimulus selectivity. J Neurosci 22:4577-4590.

Bastian J, Chacron MJ, Maler L (2004) Plastic and non-plastic cells perform unique roles in a network capable of adaptive redundancy reduction. Neuron 41:767-779.

Bell C, Maler L (2005) Central neuroanatomy of electrosensory systems in fish. In: Electroreception (Bullock TH, Hopkins CD, Popper AN, Fay RR, eds), pp 68-111. New York: Springer.

Benda J, Longtin A, Maler L (2005) Spike-frequency adaptation separates transient communication signals from background oscillations. J Neurosci 25:2312-2321.

Bertoncini J, Serniclaes W, Lorenzi C (2009) Discrimination of speech sounds based upon temporal envelope versus fine structure cues in 5- to 7-year-old children. J Speech Lang Hear Res 52:682-695.

Brenner N, Bialek W, de Ruyter van Steveninck R (2000) Adaptive rescaling maximizes information transmission. Neuron 26:695-702.

Carr CE, Maler L (1985) A Golgi study of the cell types of the dorsal torus semicircularis of the electric fish Eigenmannia: functional and morphological diversity in the midbrain. J Comp Neurol 235:207-240.

Carr CE, Maler L (1986) Electroreception in gymnotiform fish. Central anatomy and physiology. In: Electroreception (Bullock TH, Heiligenberg W, eds), pp 319-373. New York: Wiley.

Chacron MJ (2006) Nonlinear information processing in a model sensory system. J Neurophysiol 95:2933-2946.

Chacron MJ, Bastian J (2008) Population coding by electrosensory neurons. J Neurophysiol 99:1825-1835.

Chacron MJ, Fortune ES (2010) Subthreshold membrane conductances enhance directional selectivity in vertebrate sensory neurons. J Neurophysiol 104:449-462.

Chacron MJ, Doiron B, Maler L, Longtin A, Bastian J (2003) Non-classical receptive field mediates switch in a sensory neuron's frequency tuning. Nature 423:77-81.

Chacron MJ, Longtin A, Pakdaman K (2004) Chaotic firing in the sinusoidally forced leaky integrate-and-fire model with threshold fatigue. Physica D 192:138-160.

Chacron MJ, Maler L, Bastian J (2005a) Electroreceptor neuron dynamics shape information transmission. Nat Neurosci 8:673-678.

Chacron MJ, Maler L, Bastian J (2005b) Feedback and feedforward control of frequency tuning to naturalistic stimuli. J Neurosci 25:5521-5532.

Chacron MJ, Longtin A, Maler L (2005c) Delayed excitatory and inhibitory feedback shape neural information transmission. Phys Rev E 72:051917.

Chacron MJ, Lindner B, Longtin A (2007) Threshold fatigue and information transfer. J Comput Neurosci 23:301-311.

Chacron MJ, Toporikova N, Fortune ES (2009) Differences in the time course of short-term depression across receptive fields are correlated with directional selectivity in electrosensory neurons. J Neurophysiol 102:3270-3279.

Chacron MJ, Longtin A, Maler L (2011) Efficient computation via sparse coding in electrosensory neural networks. Curr Opin Neurobiol 21:752-760.

Curtis JC, Kleinfeld D (2009) Phase-to-rate transformations encode touch in cortical neurons of a scanning sensorimotor system. Nat Neurosci 12:492-501.

de Kock CP, Sakmann B (2009) Spiking in primary somatosensory cortex during natural whisking in awake head-restrained rats is cell-type specific. Proc Natl Acad Sci U S A 106:16446-16450.

Duensing F, Schaefer KP (1958) [The activity of single neurons in the region of the vestibular nuclei in horizontal acceleration, with special reference to vestibular nystagmus]. Arch Psychiatr Nervenkr Z Gesamte Neurol Psychiatr 198:225-252.

Ewert TA, Vahle-Hinz C, Engel AK (2008) High-frequency whisker vibration is encoded by phase-locked responses of neurons in the rat's barrel cortex. J Neurosci 28:5359-5368

Fairhall AL, Lewen GD, Bialek W, de Ruyter Van Steveninck RR (2001) Efficiency and ambiguity in an adaptive neural code. Nature 412:787-792.

Fairhall AL, Burlingame CA, Narasimhan R, Harris RA, Puchalla JL, Berry MJ 2nd (2006) Selectivity for multiple stimulus features in retinal ganglion cells. J Neurophysiol 96:2724-2738.

Fortune ES, Rose GJ (1997a) Passive and active membrane properties contribute to the temporal filtering properties of midbrain neurons in vivo. J Neurosci 17:3815-3825.

Fortune ES, Rose GJ (1997b) Temporal filtering properties of ampullary electrosensory neurons in the torus semicircularis of Eigenmannia: Evolutionary and computational implications. Brain Behav Evol 49:312-323.

Gelfand S (2004) Hearing: an introduction to psychological and physiological acoustics. Colchester: Informa Healthcare.

George AA, Lyons-Warren AM, Ma X, Carlson BA (2011) A diversity of synaptic filters are created by temporal summation of excitation and inhibition. J Neurosci 31:14721-14734.

Gollisch T, Meister M (2008) Modeling convergent ON and OFF pathways in the early visual system. Biol Cybern 99:263-278.

Greschner M, Thiel A, Kretzberg J, Ammermüller J (2006) Complex spikeevent pattern of transient ON-OFF retinal ganglion cells. J Neurophysiol 96:2845-2856.

Gussin D, Benda J, Maler L (2007) Limits of linear rate coding of dynamic stimuli by electroreceptor afferents. J Neurophysiol 97:2917-2929.

Heil P (2003) Coding of temporal onset envelope in the auditory system. Speech Commun 41:123-134.

Hitschfeld EM, Stamper SA, Vonderschen K, Fortune ES, Chacron MJ (2009) Effects of restraint and immobilization on electrosensory behaviors of weakly electric fish. ILAR J 50:361-372.

Hupé GJ, Lewis JE (2008) Electrocommunication signals in free swimming brown ghost knifefish, Apteronotus leptorhynchus. J Exp Biol 211:1657-1667.

Joris PX, Schreiner CE, Rees A (2004) Neural processing of amplitudemodulated sounds. Physiol Rev 84:541-577.

Kawasaki M (2005) Central neuroanatomy of electrosensory systems in fish. In: Electroreception (Bullock TH, Hopkins CD, Popper AN, Fay RR, eds), pp 154-194. New York: Springer.

Keener JP, Hoppensteadt FC, Rinzel J (1981) Integrate-and-fire model of nerve membrane response to oscillatory input. SIAM J Applied Mathematics 41:816-823.

Khosravi-Hashemi N, Fortune ES, Chacron MJ (2011) Coding movement direction by burst firing in electrosensory neurons. J Neurophysiol 106:1954-1968

Klam F, Graf W (2003) Vestibular signals of posterior parietal cortex neurons during active and passive head movements in macaque monkeys. Ann N Y Acad Sci 1004:271-282.

Kloeden PE, Platen E (1999) Numerical solutions of stochastic differential equations. Berlin: Springer.

Krahe R, Bastian J, Chacron MJ (2008) Temporal processing across multiple topographic maps in the electrosensory system. J Neurophysiol 100:852-867.

Lapicque L (1907) Recherches quantitatives sur l'excitation électrique des nerfs traitée comme une polarisation. J Physiol Pathol Gen 9:620-635.

Laughlin SB (1989) The role of sensory adaptation in the retina. J Exp Biol 146:39-62. 
Lewicki MS (2002) Efficient coding of natural sounds. Nat Neurosci 5:356-363.

Livingstone MS, Hubel DH (1987) Psychophysical evidence for separate channels for the perception of form, color, movement, and depth. J Neurosci 7:3416-3468.

Lundstrom BN, Higgs MH, Spain WJ, Fairhall AL (2008) Fractional differentiation by neocortical pyramidal neurons. Nat Neurosci 11:1335-1342.

Lundstrom BN, Fairhall AL, Maravall M (2010) Multiple timescale encoding of slowly varying whisker stimulus envelope in cortical and thalamic neurons in vivo. J Neurosci 30:5071-5077.

Maler L (1979) The posterior lateral line lobe of certain gymnotiform fish. Quantitative light microscopy. J Comp Neurol 183:323-363.

Maler L, Sas EK, Rogers J (1981) The cytology of the posterior lateral line lobe of high frequency weakly electric fish (Gymnotidae): differentiation and synaptic specificity in a simple cortex. J Comp Neurol 195:87-139.

Maler L, Sas E, Johnston S, Ellis W (1991) An atlas of the brain of the weakly electric fish Apteronotus leptorhynchus. J Chem Neuroanat 4:1-38.

Mante V, Frazor RA, Bonin V, Geisler WS, Carandini M (2005) Independence of luminance and contrast in natural scenes and in the early visual system. Nat Neurosci 8:1690-1697.

Maravall M, Petersen RS, Fairhall AL, Arabzadeh E, Diamond ME (2007) Shifts in coding properties and maintenance of information transmission during adaptation in barrel cortex. PLoS Biol 5:e19.

Mardia KV, Jupp PE (1999) Directional statistics. New York: Wiley.

Marr D (1982) Vision. New York: Freeman.

Mehaffey WH, Fernandez FR, Maler L, Turner RW (2007) Regulation of burst dynamics improves differential encoding of stimulus frequency by spike train segregation. J Neurophysiol 98:939-951.

Merigan WH, Maunsell JH (1993) How parallel are the primate visual pathways? Annu Rev Neurosci 16:369-402.

Middleton JW, Longtin A, Benda J, Maler L (2006) The cellular basis for parallel neural transmission of a high-frequency stimulus and its lowfrequency envelope. Proc Natl Acad Sci U S A 103:14596-14601.

Middleton JW, Yu N, Longtin A, Maler L (2011) Routing the flow of sensory signals using plastic responses to bursts and isolated spikes: experiment and theory. J Neurosci 31:2461-2473.

Myers LJ, Lowery M, O’Malley M, Vaughan CL, Heneghan C, St Clair Gibson A, Harley YX, Sreenivasan R (2003) Rectification and non-linear preprocessing of EMG signals for cortico-muscular analysis. J Neurosci Methods 124:157-165.

Nagel KI, Doupe AJ (2006) Temporal processing and adaptation in the songbird auditory forebrain. Neuron 51:845-859.

Oertel D (1999) The role of timing in the brain stem auditory nuclei of vertebrates. Annu Rev Physiol 61:497-519.

Partridge BL, Heiligenberg W, Matsubara J (1981) The neural basis of a sensory filter in the jamming avoidance response: no grandmother cells in sight. J Comp Physiol A Neuroethol Sens Neural Behav Physiol 145:153-168.

Roddey JC, Girish B, Miller JP (2000) Assessing the performance of neural encoding models in the presence of noise. J Comput Neurosci 8:95-112.
Rose GJ, Call SJ (1992) Evidence for the role of dendritic spines in the temporal filtering properties of neurons: the decoding problem and beyond. Proc Natl Acad Sci U S A 89:9662-9665.

Rose GJ, Call SJ (1993) Temporal filtering properties of midbrain neurons in an electric fish: implications for the function of dendritic spines. J Neurosci 13:1178-1189.

Rose GJ, Fortune ES (1996) New techniques for making whole-cell recordings from CNS neurons in vivo. Neurosci Res 26:89-94.

Saunders J, Bastian J (1984) The physiology and morphology of two classes of electrosensory neurons in the weakly electric fish Apteronotus Leptorhynchus. J Comp Physiol A Neuroethol Sens Neural Behav Physiol 154:199-209.

Savard M, Krahe R, Chacron MJ (2011) Neural heterogeneities influence envelope and temporal coding at the sensory periphery. Neuroscience 172:270-284.

Scheich H, Bullock TH, Hamstra RH Jr (1973) Coding properties of two classes of afferent nerve fibers: high frequency electroreceptors in the electric fish, Eigenmania. J Neurophysiol 36:39-60.

Schiller PH, Sandell JH, Maunsell JH (1986) Functions of the ON and OFF channels of the visual system. Nature 322:824-825.

Schneider AD, Cullen KE, Chacron MJ (2011) In vivo conditions induce faithful encoding of stimuli by reducing nonlinear synchronization in vestibular sensory neurons. PLoS Comput Biol 7:e1002120.

Shannon RV, Zeng FG, Kamath V, Wygonski J, Ekelid M (1995) Speech recognition with primarily temporal cues. Science 270:303-304.

Shannon RV, Zeng FG, Wygonski J (1998) Speech recognition with altered spectral distribution of envelope cues. J Acoust Soc Am 104:2467-2476.

Simoncelli EP, Olshausen BA (2001) Natural image statistics and neural representation. Annu Rev Neurosci 24:1193-1216.

Slee SJ, Higgs MH, Fairhall AL, Spain WJ (2005) Two-dimensional time coding in the auditory brainstem. J Neurosci 25:9978-9988.

Stamper SA, Carrera GE, Tan EW, Fugère V, Krahe R, Fortune ES (2010) Species differences in group size and electrosensory interference in weakly electric fishes: implications for electrosensory processing. Behav Brain Res 207:368-376.

Svirskis G, Dodla R, Rinzel J (2003) Subthreshold outward currents enhance temporal integration in auditory neurons. Biol Cybern 89:333-340.

Takahashi T, Moiseff A, Konishi M (1984) Time and intensity cues are processed independently in the auditory system of the owl. J Neurosci 4:1781-1786.

Toporikova N, Chacron MJ (2009) Dendritic SK channels gate information processing in vivo by regulating an intrinsic bursting mechanism seen in vitro. J Neurophysiol 102:2273-2287.

Trussell LO (1999) Synaptic mechanisms for coding timing in auditory neurons. Annu Rev Physiol 61:477-496.

Vonderschen K, Chacron MJ (2011) Sparse and dense coding of natural stimuli by distinct midbrain neuron subpopulations. J Neurophysiol 106:3102-3118.

Wark B, Lundstrom BN, Fairhall A (2007) Sensory adaptation. Curr Opin Neurobiol 17:423-429. 